\title{
Influence of Temperature on the Electrical Characteristic Parameters and Dynamic Electromechanical Behaviour of PZT-5H
}

Enling Tang ( $\square$ tangenling@126.com )

Shenyang Ligong University

Hetong Yin

Shenyang Ligong University

Yafei Han

Shenyang Ligong University

Chuang Chen

shenyang ligong university

\section{Research Article}

Keywords: Temperature effect; PZT-5H; Electrical parameters; Electromechanical behaviour; Freezing effect

Posted Date: May 11th, 2020

DOl: https://doi.org/10.21203/rs.3.rs-27161/v1

License: (9) This work is licensed under a Creative Commons Attribution 4.0 International License. Read Full License 


\title{
Influence of Temperature on the Electrical Characteristic Parameters and Dynamic Electromechanical Behaviour of PZT-5H
}

\author{
Enling Tang*, Hetong Yin, Yafei Han*, Chuang Chen \\ (Intense Dynamic Load Research Center, Shenyang ligong University, Shenyang 110159 China) \\ *Corresponding author. E-mail addresses: tangenling@126.com; hanyafei1979@126.com
}

\begin{abstract}
In order to study the electromechanical characteristics of PZT-5H in the temperature range of $-40 \sim 25^{\circ} \mathrm{C}$ under the impact loading, the electrostatic parameters of PZT-5H at different temperatures are measured, the liquid nitrogen cooling/Hopkinson mechanical property and piezoelectric ceramic voltage output testing system are built, Hopkinson experiments at different temperatures $\left(-40{ }^{\circ} \mathrm{C},-25^{\circ} \mathrm{C},-10{ }^{\circ} \mathrm{C}, 0{ }^{\circ} \mathrm{C}\right.$ and $\left.25^{\circ} \mathrm{C}\right)$ and different impact velocities are carried out. Experimental results show that, the resistivity and relative permittivity decrease with the temperature of PZT-5H decreasing from $25^{\circ} \mathrm{C}$ to $-40^{\circ} \mathrm{C}$, and the relative permittivity decrease about $27.92 \%$, and the relaxation time is also deduced. By scanning electron microscope (SEM) and Energy Dispersive Spectrometer (EDS) observation, it is found that the blowhole defects and cracks of the piezoelectric ceramics have obvious effects on the electrical output characteristics of piezoelectric ceramics when the piezoelectric ceramics are struck. Based on the analysis of space charge theory, the freezing effect of piezoelectric ceramics caused by the obstruction of domain motion at low temperature is obtained. And the dynamic piezoelectric constant in the elastic region measured is about 1.66 times that of the static piezoelectric constant, which indicates that the relative dielectric constant of the piezoelectric ceramic has changed significantly under dynamic loading.
\end{abstract}

Keywords: Temperature effect; PZT-5H; Electrical parameters; Electromechanical behaviour; Freezing effect

\section{Introduction}

Since 1954, Jaffe et $\mathrm{al}^{[1]}$. had made PZT ferroelectric ceramics for the first time, many scholars had carried out a lot of experimental research on the properties of PZT series ceramics. In 1960, McKinney et al ${ }^{[2]}$. carried out $0-100 \mathrm{Mpa}$ pressure tests on the hybrid ferroelectric system near room temperature $\left(25-37.5^{\circ} \mathrm{C}\right)$, and analyzed the piezoelectric and dielectric properties of the ferroelectric materials for the first time under the thermal mechanical coupling. In 1994, Qiming Zhang and et $\mathrm{a}^{[3]}$. introduced an experimental method for directly and quantitatively measuring the domain walls and intrinsic contributions of piezoelectric and dielectric response of ferroelectric materials, and evaluated the contribution of domain walls and intrinsic parts of PZT 52/48 and PZT-500 ceramics and their relationship with temperature by using this method. In 1995, Noheda et $a^{[4]}$. compared the phase transition temperature of PZT ferroelectric ceramics with 91-97\% zirconium content under two conditions of heating and cooling, and studied the hysteresis phenomenon of phase transition of ferroelectric materials with different loading ways and different zirconium titanium ratio. In 1998, Hooker ${ }^{[5]}$ measured the dielectric, ferroelectric and piezoelectric properties of PZT-4, PZT-5A, PZT-5H and PLZT-9/65/35 in the temperature range of $-150 \sim 250{ }^{\circ} \mathrm{C}$. The results showed that PZT-4 was relatively stable in the temperature range of $-150{ }^{\circ} \mathrm{C}$ to $25{ }^{\circ} \mathrm{C}$, and PZT-5H had better piezoelectric properties at room temperature. The physical mechanism of the influence of low temperature on the dielectric, ferroelectric and piezoelectric properties of PZT-5H was not clarified. In 2002, Panda ${ }^{[6]}$ carried out thermal shock fatigue test on PZT-5 piezoelectric ceramics by keeping the temperature of both sides of the sample constant and having a large temperature difference, and then generated the temperature gradient inside the material to simulate the impact of thermal shock on the ferroelectric ceramics. In 2013, Andryushina et $\mathrm{al}^{[7]}$. revealed the relationship between the relaxation process of $\mathrm{PbTi}_{0.495} \mathrm{Zr}_{0.505} \mathrm{O}_{3}$ solid solution and the change of structural characteristics in the temperature range of $100-300 \mathrm{~K}$. This study only explained the change rule of temperature on the structural characteristics of PZT based ceramics, and did not discuss its electrical characteristics and 
electromechanical coupling characteristics. In the same year, Weijiang Xue et al ${ }^{[8]}$. studied the transformation mechanism and performance of Zirconia based structural ceramics at low temperature, the low temperature performance of alumina ceramics, the basic mechanical properties and fracture mechanism of Silicon Nitride, Silicon Carbide and other Non Oxide ceramics at low temperature. But in this experiment, the mechanical properties and fracture mechanism of structural ceramics were analyzed only under quasi-static condition, and the dynamic properties at low temperature were not studied. Lan $\mathrm{C} \mathrm{F}$ et al ${ }^{[0]}$. revealed the change rule of phase structure and electrical properties of dense PZT95/5 Ferroelectric Ceramics at low temperature, but did not further explain the micro change of phase structure and electrical properties of dense PZT95/5 ferroelectric ceramics under low temperature. In 2016, Anand et al ${ }^{[10]}$. proposed a temperature dependent micromechanical model based on internal energy to evaluate the ferroelectric and ferroelastic behavior of PZT wafers, extracted macro state variables (residual polarization, residual strain, maximum polarization and maximum strain, etc.) and discussed them as a function of temperature. Cao $\mathrm{W}$ Q et $\mathrm{al}^{[11]}$ applied the phenomenological theory of Ferroelectrics and the response of electric domain to alternating electric field, and put forward a model of the hysteresis freezing effect of the domain in the measurement of hysteresis loop, and explained reasonably the phenomenon of polarization degradation at low temperature. In 2018, Belovickis et al ${ }^{[12]}$.studied the dielectric spectrum, ferroelectric and piezoelectric properties of $\mathrm{P}(\mathrm{VDF}-\mathrm{TrFE}) / \mathrm{BPZT}$ in the temperature range of $150-420 \mathrm{~K}$, and analyzed the relationship between the effective dielectric constant of the composite and the filler concentration by Lichtenecker method. Tang E L et al ${ }^{[13-17]}$. carried out a large number of dynamic mechanical and electrical performance studies on PZT-5H from 2014 to 2019, and obtained the mechanical response and piezoelectric characteristics of the piezoelectric ceramics under different strain rates, and evaluated the electrical output characteristics of the laminated piezoelectric ceramics under overload conditions, and the influence mechanism of the internal cracks on the discharge characteristics of the ceramics were studied. In 2020, Flannigan et al ${ }^{[18]}$. found that the energy index of PZT was strongly dependent on temperature, and evolved into a superposition value of about 2.0 with the change of temperature. Wang et al ${ }^{[19]}$. studied the temperature dependence of PZT-5H piezoelectric properties. The experimental results show that the piezoelectric strain coefficients of PZT-5H changes linearly with temperature, the decrease of temperature leads to the decrease of the measured piezoelectric coefficients. The internal mechanical stresses is the main mechanism of PZT-5H ferroelectric fatigue, and a set of enhanced constitutive relation which can be used to extrapolate some nonlinear characteristics to other temperature regions is established.

At present, there are many researches on the electrostatic properties of piezoelectric ceramics at room temperature, but the dynamic mechanical-electrical response characteristics of piezoelectric ceramics at low temperature are yet not clear. In this paper, the resistivity and relative permittivity of PZT-5H in the temperature range of $-40 \sim 25$ are measured, and Hopkinson pressure bar experiments are performed for PZT-5H at the temperature of $-40,-25,-10,0$ and $25^{\circ} \mathrm{C}$ and different impact velocities. Besides, the fracture and the mechanical-electrical response characteristics of PZT-5H at different temperatures and impact velocities are analyzed based on the theory of one-dimensional elastic stress wave propagation and microscopic observation.

\section{Materials and methods}

\subsection{Materials}

The PZT-5H $\left(\mathrm{Pb}_{1.0}\left[\mathrm{Zr}_{0.49} \mathrm{Ti}_{0.46}\left(\mathrm{La}_{0.25} \mathrm{Sb}_{0.5} \mathrm{Fe}_{0.25}\right)_{0.05}\right] \mathrm{O}_{3}\right)$ cylindrical piezoelectric ceramic with 
a diameter of $\Phi 14 \times 3 \mathrm{~mm}^{2}$ produced by Yuhai Electronic Ceramics Co., Ltd, China, is used in experiment. Fig. 1 lists the physical diagram of PZT-5H. The basic performance parameters of PZT-5H at $25^{\circ} \mathrm{C}$ are shown in Table 1.

Table1 Basic performance parameters of PZT-5H $\left(25^{\circ} \mathrm{C}\right)$

\begin{tabular}{cccccc}
\hline Density $/ \mathrm{kg} \cdot \mathrm{m}^{-3}$ & $\begin{array}{c}\text { Curie } \\
\text { point } /{ }^{\circ} \mathrm{C}\end{array}$ & Mechanical $\mathrm{Q}_{\mathrm{m}}$ & $\begin{array}{c}\text { Acoustic } \\
\text { velocity } / \mathrm{m} \cdot \mathrm{s}^{-1}\end{array}$ & $\begin{array}{c}\text { Young's } \\
\text { modulus } / 10^{9} \mathrm{~N} \cdot \mathrm{m}^{2}\end{array}$ & Poisson's ratio \\
\hline 7500 & 230 & 0.76 & 3100 & 56 & 0.36 \\
\hline
\end{tabular}

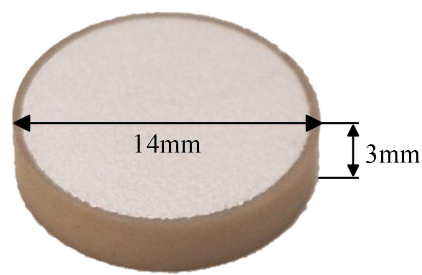

\subsection{Experimental method}

Fig. 1 The physical diagram of PZT-5H

PZT-5H static tests have been conducted in TH2832 LCR digital tester of Changzhou Tonghui Electronic Co., Ltd, China. The low temperature PZT-5H experiments at different impact velocities were carried out on the separated Hopkinson pressure bar experimental system in the intense dynamic load research center of Shenyang Ligong University, China. Fig. 2 is the schematic diagram of the experimental system.

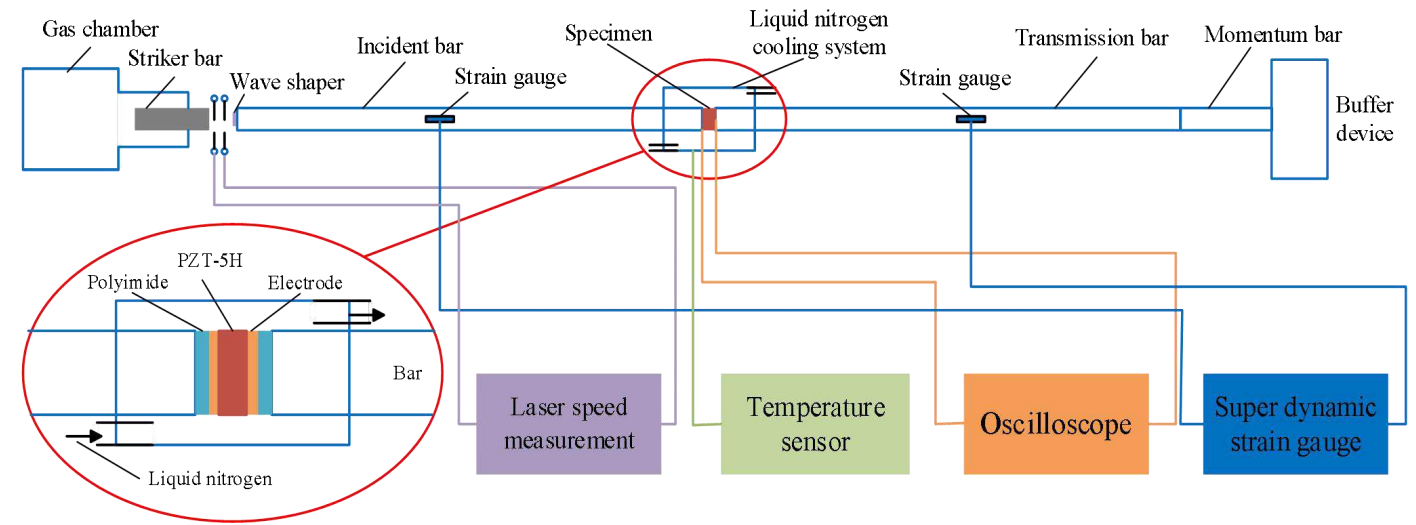

Fig. 2 The schematic diagram of the experimental system

The conductive copper foil is used to connect the positive and negative electrodes of the ceramic. The output voltage at both ends of the electrode is collected by the voltage probe acquisition system and recorded by the oscilloscope to obtain the time history curve of the voltage pulse. To prevent electrical breakdown, the polyimide film is used for insulation treatment at both ends of the specimen.

In order to carry out experiments at low temperature, the liquid nitrogen cooling system of low temperature experiment tank is designed, the liquid nitrogen is injected into the tank and outflowed to the outside to reduce the internal temperature of the tank, the temperature in the tank is controlled by controlling the flow rate of liquid nitrogen, and the temperature of the specimen is monitored by the thermocouple temperature sensor. In order to prevent the specimen from being affected by temperature difference, the rate of controlling the temperature change of the specimen is not more than $3{ }^{\circ} \mathrm{C} / \mathrm{min}$; in order to ensure the constant temperature of the Hopkinson pressure bar, the pressure bar does not hold the test specimen at this time. After the temperature of the test 
specimen is stable (the temperature change is less than $2{ }^{\circ} \mathrm{C}$ ) to more than 15 minutes, the test specimen is clamped between the incidence bar and the transmission bar of the Hopkinson experimental system.

According to one-dimensional elastic stress wave theory ${ }^{[20]}$, the strain rate $\dot{\varepsilon}$, strain $\varepsilon$ and stress $\sigma$ of the specimen can be calculated directly under the assumption of one-dimensional stress loading and uniform distribution of stress and strain along its length

$$
\begin{gathered}
\dot{\varepsilon}=\frac{c_{0}}{l_{0}}\left(\varepsilon_{1}-\varepsilon_{2}-\varepsilon_{3}\right) \\
\varepsilon=\frac{c_{0}}{l_{0}} \int_{0}^{t}\left(\varepsilon_{1}-\varepsilon_{2}-\varepsilon_{3}\right) \mathrm{d} \tau \\
\sigma=\frac{A E}{2 A_{0}}\left(\varepsilon_{1}+\varepsilon_{2}+\varepsilon_{3}\right)
\end{gathered}
$$

In formulas (1) (3), $c_{0}$ is the elastic wave velocity in the bar, $\varepsilon_{1}, \varepsilon_{2}$ and $\varepsilon_{3}$ are the strains in the bar corresponding to the incident wave, the reflected wave and the transmitted wave when they propagate independently. $l_{0}$ and $A_{0}$ are the original length and cross-sectional area of the specimen respectively. $A$ and $E$ are the cross-sectional area and elastic modulus of the bar, respectively. The duration of the stress pulse on the specimen is equal to the time of the wave going back and forth once in the impact bar ${ }^{[20]}$, which can be expressed as

$$
t=\frac{2 L}{c_{0}}
$$

In formula (4), $L$ is the length of impact bar. It can be known that the loading frequency of the specimen is $\omega_{0}=1 / t_{0}=17.75 \mathrm{kHz}$.

\section{The influence of temperature on electrostatic characteristic parameters of PZT-5H}

\subsection{Resistivity}

The resistivities of PZT-5H at different temperatures are measured by TH2832 LCR digital tester. Fig. 3 shows the spectrum of PZT-5H resistivity at different temperatures. Fig. 4 is the relationship between resistivity and temperature of PZT-5H at loading frequency $\omega_{0}$.

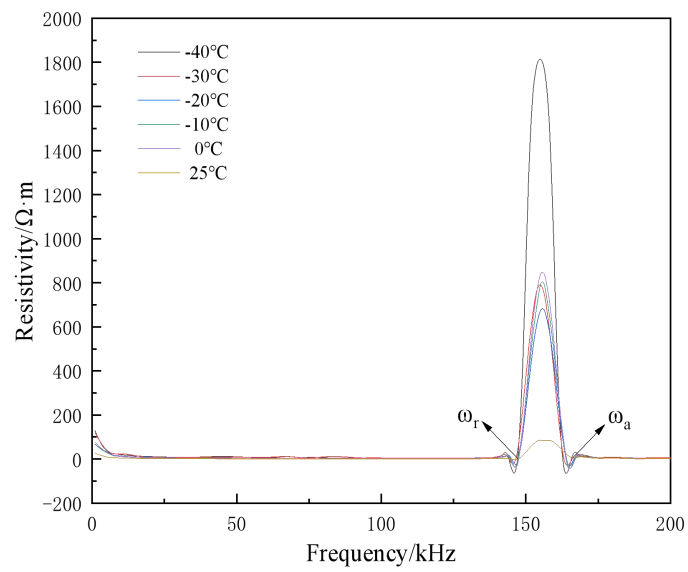

Fig. 3 The resistivity spectrum of PZT-5H at different temperatures

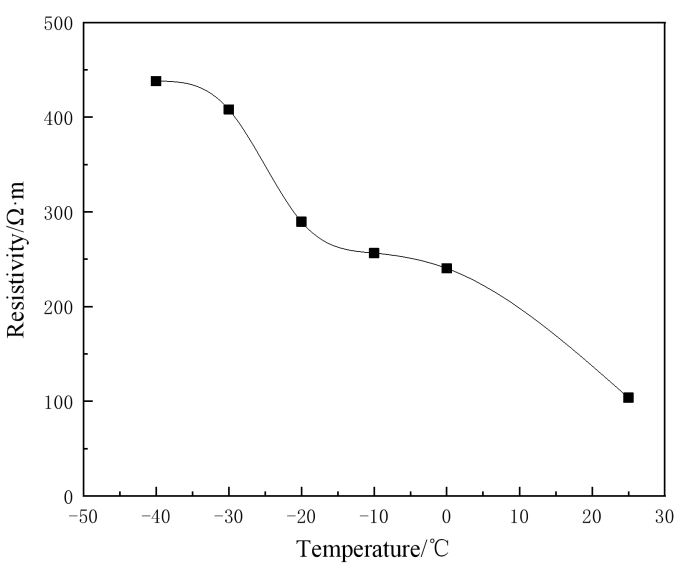

Fig. 4 The variation of resistivity with temperature under loading frequency $\omega_{0}$

The piezoelectric crystal is an impedance component with resonant frequency $\omega_{r}$ and 
anti-resonant frequency $\omega_{a}$, when the loading frequency on the piezoelectric crystal is equal to its resonance frequency $\omega_{r}$, the piezoelectric crystal will resonate. At this time, the impedance angle is the maximum value in the negative direction and the resistance is the minimum value of the impedance angle; when the loading frequency increases to the frequency with the impedance angle of 0 , the resistance has a peak value; when the loading frequency of the piezoelectric crystal increases to the anti-resonance frequency $\omega_{a}$, the impedance angle increases to the maximum value of the forward direction, and the resistance is the maximum value of the impedance angle.

As can be seen from Fig. 3, at the temperature range of $-40^{\circ} \mathrm{C} \sim 25^{\circ} \mathrm{C}$, the resonant frequency of PZT-5H is $147.359 \mathrm{kHz}$, and the anti-resonant frequency is $163.931 \mathrm{kHz}$. The influence of the frequency between $\omega_{r}$ and $\omega_{a}$ on the resistivity of PZT-5H is obvious and the peak appears. Fig. 4 shows that the resistivity of PZT-5H decreases with the increase of temperature at this frequency, but the decrease of resistivity above $0^{\circ} \mathrm{C}$ is steeper than that below $0^{\circ} \mathrm{C}$.

In theory, the intrinsic conductance of electrons as carriers and the non intrinsic conductance of ions in dielectric materials, ion clusters and space charges as carriers determine the resistivity of materials ${ }^{[21]}$. Conductivity $\sigma_{e}$, the number $n$ and mobility of conduction electrons $u$ per unit volume are satisfied with

$$
\sigma_{e}=\frac{1}{\rho}=n e u
$$

At room temperature $\left(25^{\circ} \mathrm{C}\right)$, the carrier is mainly excited by electrons from full band to conduction band, that is, the intrinsic excitation of piezoelectric ceramics. At low temperatures, the carrier is mainly excited by electrons from the impurity donor to the conduction band, that is, the impurity excitation of the piezoelectric ceramics. The intrinsic excitation and impurity excitation of PZT-5H can increase the conduction electron with the increase of temperature. However, the energy difference produced by intrinsic excitation is much larger than that produced by impurity excitation ${ }^{[21]}$. Therefore, the change trend of intrinsic excitation with temperature is more steep, which is consistent with the conclusion in literature [5].

\subsection{Dielectric properties}

Dielectric property is the most basic physical property of piezoelectric ceramics. Because of the loss in piezoelectric ceramic measurement, the relative permittivity $\varepsilon_{r}$ of piezoelectric ceramics can be expressed in the form of complex number as

$$
\varepsilon_{r}^{*}(\omega)=\varepsilon_{r}^{\prime}(\omega)-j \varepsilon_{r}^{\prime \prime}(\omega)
$$

In formula $(6), \varepsilon_{r}^{\prime}(\omega)$ is the real part of relative complex permittivity under alternating electric field, and $\varepsilon_{r}^{\prime \prime}(\omega)$ is the imaginary part of the relative complex permittivity, representing the dielectric loss. Fig. 5 shows the relative permittivity spectrum of PZT-5H at different temperatures. Fig. 6 shows the dielectric loss spectrum of PZT-5H at different temperatures. 


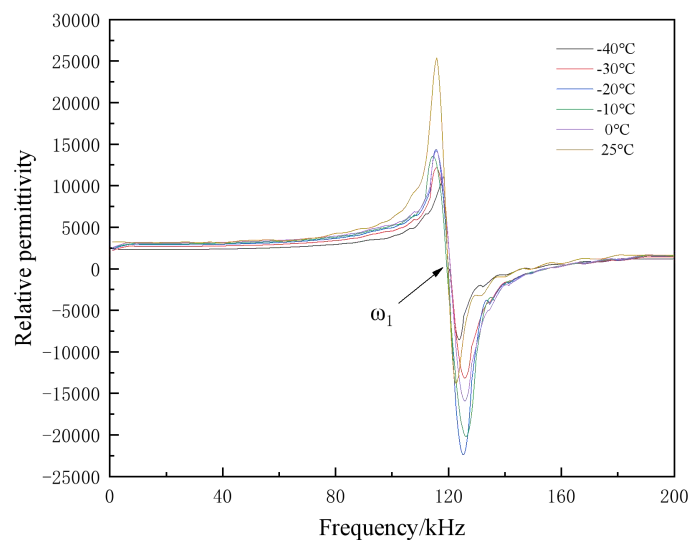

Fig. 5 The relative permittivity spectrum of PZT-5H at different temperatures

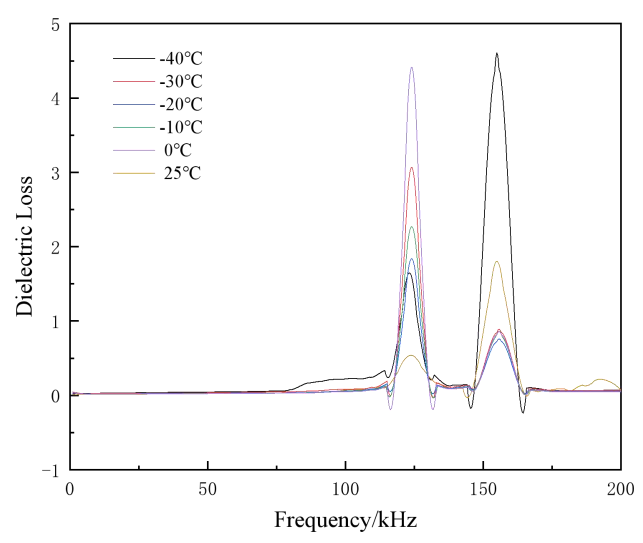

Fig. 6 The dielectric loss spectrum of PZT-5H at different temperatures

As can be seen from Fig. 5 , in the temperature range of $40^{\circ} \mathrm{C} \sim 25^{\circ} \mathrm{C}$, the dielectric constant of PZT-5H has obvious differences near the vibration frequency $\omega_{1}\left(\varepsilon_{r}^{\prime}\left(\omega_{1}\right)=0\right.$ and $\omega_{1}$ is the maximum point), and resonance absorption occurs ${ }^{[23]}$, which indicates that the energy dissipation of elastic domains in the electric field is mainly near $\omega_{1}$.

Dielectric loss refers to the phenomenon that the dielectric is heated by the external electric field due to the part of consumption of the electric energy. The tangent value of dielectric loss angle shown in equation (7) can usually be expressed as

$$
\tan \delta(\omega)=\varepsilon_{r}^{\prime \prime} / \varepsilon_{r}^{\prime}
$$

Dielectric loss is generally in the form of polarization loss, leakage loss, ionization loss and structure loss. Polarization loss is closely related to frequency, and structure loss is closely related to the internal tightness of the medium at low temperature. Under the action of alternating electric field, the relation between dielectric behavior and frequency of ceramics is revealed according to Debye's equation (8) (10)

$$
\begin{gathered}
\varepsilon_{r}^{\prime}(\omega)=\varepsilon_{r \infty}+\frac{\varepsilon_{r s}-\varepsilon_{r \infty}}{1+\omega^{2} \tau^{2}} \\
\varepsilon_{r}^{\prime \prime}(\omega)=\left(\varepsilon_{r s}-\varepsilon_{r \infty}\right) \frac{\omega \tau}{1+\omega^{2} \tau^{2}} \\
\tan \delta=\frac{\left(\varepsilon_{r s}-\varepsilon_{r \infty}\right) \omega \tau}{\varepsilon_{r s}+\varepsilon_{r \infty} \omega^{2} \tau^{2}}
\end{gathered}
$$

Where, $\varepsilon_{r s}$ is the relative dielectric constant under static state, $\varepsilon_{r \infty}$ is the relative dielectric constant under optical frequency, $\tau$ is the relaxation time. It can be seen from Figs. 5 and 6 combined with formula (8) (10), when the applied frequency is very low $(\omega \rightarrow 0)$, the polarization energy can keep up with the change of the electric field, that is to say, all forms of polarization can be completely established and $\varepsilon_{r}^{\prime}$ reaches the maximum values without loss. With the increase of frequency, the relaxation polarization can not keep up with the change of electric field frequency $\omega_{1}$, and resulting in the dielectric constant decreases and $\varepsilon_{r}^{\prime}$ obviously decreases, however $\varepsilon_{r}^{\prime \prime}$ and $\tan \delta$ increase, which results in dielectric loss. When the condition is $0.01<\omega \tau<100$, the dielectric loss of piezoelectric ceramics is generated along with the energy loss. Where, $\tan \delta$ reaches peak value when $\omega$ is satisfied with $\omega=1 / \tau$ or $\omega=\sqrt{\varepsilon_{r s} / \varepsilon_{r o \infty}} / \tau$. When 
the frequency is very high, the relaxation polarization is not enough to establish and has no contribution to the dielectric constant. At this time, the dielectric constant is determined only by the displacement polarization. When the condition is $\varepsilon_{r}^{\prime} \rightarrow \varepsilon_{r \infty}$, it tends to the minimum value, and the $\varepsilon_{r}^{\prime \prime}$ and $\tan \delta$ decrease. When the conditions are $\omega \rightarrow \infty, \varepsilon_{r}^{\prime \prime} \rightarrow 0$ and $\tan \delta \rightarrow 0$, there is no dielectric loss.

Among them, relaxation time $\tau$ is a constant independent of time but related to temperature, which means that $\varepsilon_{r}^{\prime}, \varepsilon_{r}^{\prime \prime}$ and $\tan \delta$ are also closely related to temperature. According to the peak point of $\tan \delta$ in Fig. 6 , the relaxation time $\tau$ of PZT-5H at different temperatures can be deduced. Table 2 shows the relaxation time $\tau$ of PZT-5H at different temperatures.

Table 2 The relaxation time $\tau$ of PZT-5H at different temperatures

\begin{tabular}{ccccccc}
\hline $\mathrm{T} /{ }^{\circ} \mathrm{C}$ & -40 & -30 & -20 & -10 & 0 & 25 \\
\hline$\tau / \mu \mathrm{s}$ & 8.154411945 & 8.127966708 & 8.101692444 & 8.075587499 & 8.04971504 & 8.023943447 \\
\hline \multicolumn{7}{c}{ Based on the equations (8) $\sim(10)$, the relaxation time can be expressed as } \\
$\ln \tau=\mathrm{c}+\omega / \mathrm{KT}$ & $(11)$
\end{tabular}

In equation (11), $K$ and $\mathrm{C}$ are diffusion coefficient and constant. With the increase of temperature, the relaxation time decreases exponentially. The Debye's theory hypothesis shows that molecular thermal motion is the main cause of dipole inversion. The action of electric field only changes the direction of dipole turning to electric field. Fig. 7 shows the change curve of the relative dielectric constant $\varepsilon_{r}^{\prime}$ and dielectric loss of PZT-5H with temperature under the loading frequency $\omega_{0}$.

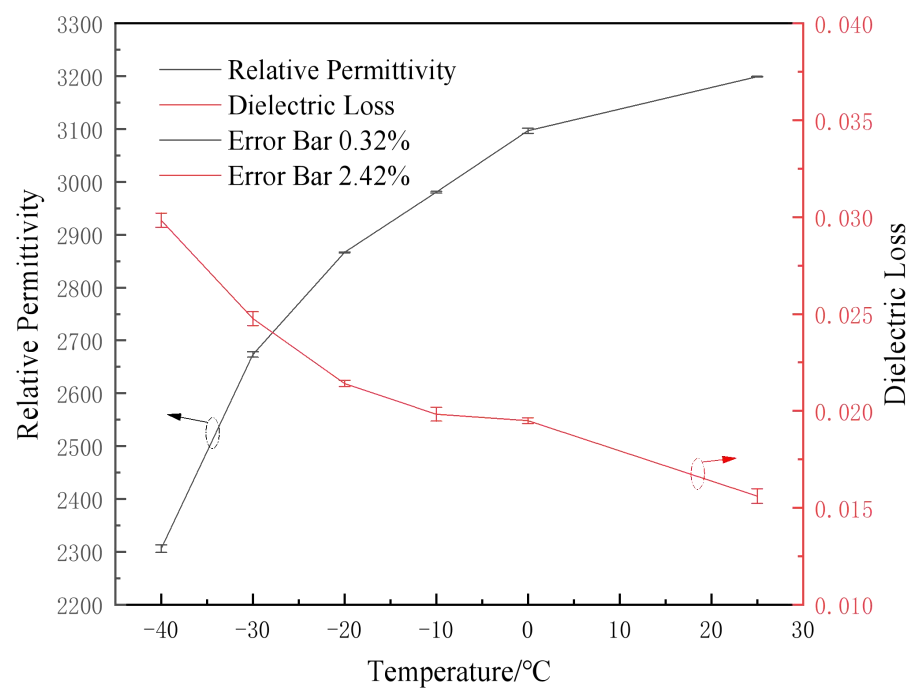

Fig. 7 The curves of relative permittivity $\varepsilon_{r}^{\prime}$, dielectric loss and temperature at loading frequency $\omega_{0}$

At room temperature, the relative permittivity of PZT-5H is 3199.3. With the decrease of temperature, the relative permittivity curve decreases to 2306.1 at the temperature of $-40{ }^{\circ} \mathrm{C}$. Compared with the temperature of $25^{\circ} \mathrm{C}$, the relative permittivity of PZT-5H decreases by $27.92 \%$ at the temperature of $-40{ }^{\circ} \mathrm{C}$, but the corresponding loss increased from 0.0156 at the temperature of $25^{\circ} \mathrm{C}$ to 0.03 at the temperature of $-40^{\circ} \mathrm{C}$. In Fig. 7, the dielectric constant of PZT-5H has no mutation and the loss has no peak value, indicating that PZT-5H has no phase transition in this temperature range ${ }^{[24]}$. The results are consistent with that of PZT95/5 ferroelectric ceramics in reference [9]. 
In the normal coordinate system, the relative permittivity of $\mathrm{N}$-square lattice ceramics is related to the lattice vibration frequency. Anderson ${ }^{[24]}$ points out that the effective Hamiltonian can be used to qualitatively explain the dielectric anomaly. When the propagation vector is $\beta=0$, the effective Hamiltonian of the potential energy of the lattice optical oscillator is related to the coefficient of thermal expansion. According to the Slater-Devonshire theory ${ }^{[25]}$, using the relationship between relative permittivity and lattice vibration frequency and the equilibrium condition of potential energy effective Hamiltonian of lattice oscillator, combining with the relative permittivity in the permittivity equation $\alpha=\left(\varepsilon_{0} \varepsilon_{\mathrm{r}}-1\right) / 4 \pi\left(\varepsilon_{0}=8.85 \times 10^{-12} \mathrm{~F} / \mathrm{m}\right.$ is the vacuum permittivity), it can be obtained that the change of relative permittivity and temperature is related to the coefficient of thermal expansion, i.e. to the effect of thermal expansion ${ }^{[26]}$, the low temperature leads to no more intense thermal motion. The smaller the expansion effect of the lattice decreases with the temperature, the closer the lattice is, the more difficult the domain rotation is, resulting in the decrease of dielectric constant with decreasing temperature.

\section{Influence of temperature on the dynamic response characteristics of PZT-5H}

At different temperatures $\left(-40{ }^{\circ} \mathrm{C},-25^{\circ} \mathrm{C},-10{ }^{\circ} \mathrm{C}, 0{ }^{\circ} \mathrm{C}\right.$ and $\left.25^{\circ} \mathrm{C}\right)$, PZT-5H impact tests are carried out at three different impact speeds $(2.4 \mathrm{~m} / \mathrm{s}, 3.4 \mathrm{~m} / \mathrm{s}$ and $4.5 \mathrm{~m} / \mathrm{s}$ (the specimen breaks at this time)).

\subsection{Influence of temperature and impact load on deformation process of PZT-5H}

Fig. 8 shows the stress and strain curve of PZT-5H at different impact speeds at the temperature of $0^{\circ} \mathrm{C}$.

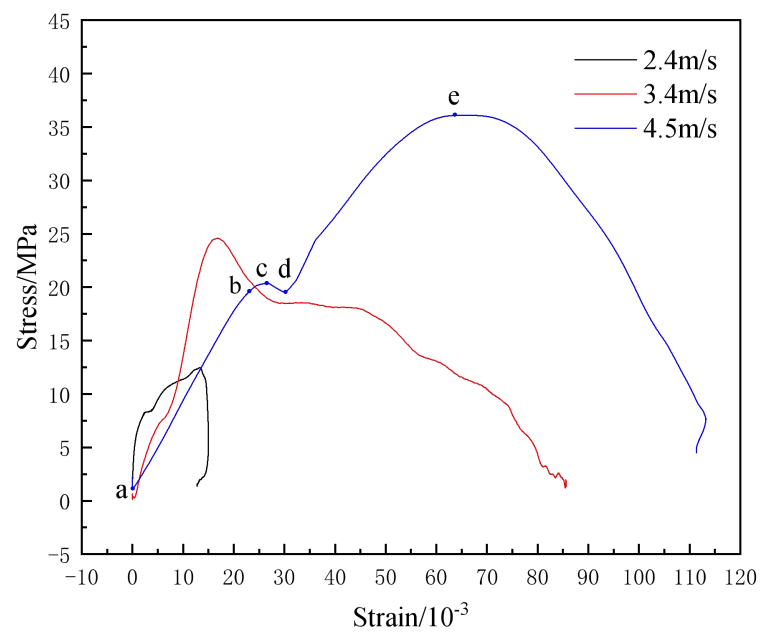

Fig. 8 The stress and strain curve of PZT-5H at different impact speeds at the temperature of $0{ }^{\circ} \mathrm{C}$

By observing the recovered post impact piezoelectric ceramic specimen, it is known that when the impact velocity is $2.4 \mathrm{~m} / \mathrm{s}$, PZT-5H does not occur macro fracture and has no obvious change. When the impact velocity is $4.5 \mathrm{~m} / \mathrm{s}$, the linear elastic deformation starts from point "a" to point " $\mathrm{b}$ " and then enters the non-elastic stage. The yield stage is in the c-d segment, and the yield point " $\mathrm{d}$ " is in the segment, and then entering the plastic hardening stage of point "e" (the fracture strength is $36.19 \mathrm{MPa}$ ). At this time, the deformation of the piezoelectric ceramics is straight to the complete fracture. Fig. 9 shows the fracture of piezoelectric ceramics. 


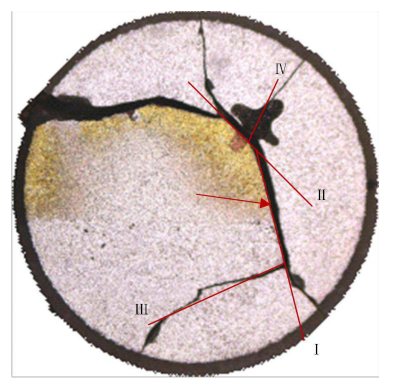

Fig. 9 The fracture of piezoelectric ceramics

It can be seen from Fig. 9 that the piezoelectric ceramics fracture along the main cracks I and II, then the cracks bifurcate and form cracks III and IV, and forming penetrating cracks along the polarization direction. Fig. 10 is a local SEM image of the fracture surface at the red arrow in the piezoelectric ceramics of Fig. 9.
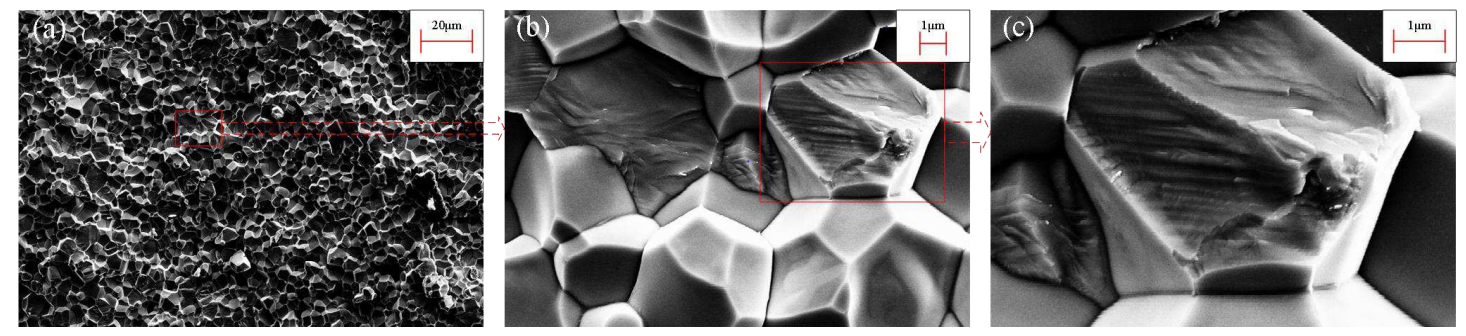

Fig. 10 Local SEM of fracture surface of piezoelectric ceramics (a) $500 \times$, (b) $5000 \times$, (c) $5000 \times$

Fig. 10 shows that PZT-5H is a cubic crystal structure. Fig. 11 shows the yield strength at impact speeds of $2.4 \mathrm{~m} / \mathrm{s}$ and $3.4 \mathrm{~m} / \mathrm{s}$, and the relationship between the fracture strength and temperature at impact speeds of $4.5 \mathrm{~m} / \mathrm{s}$.

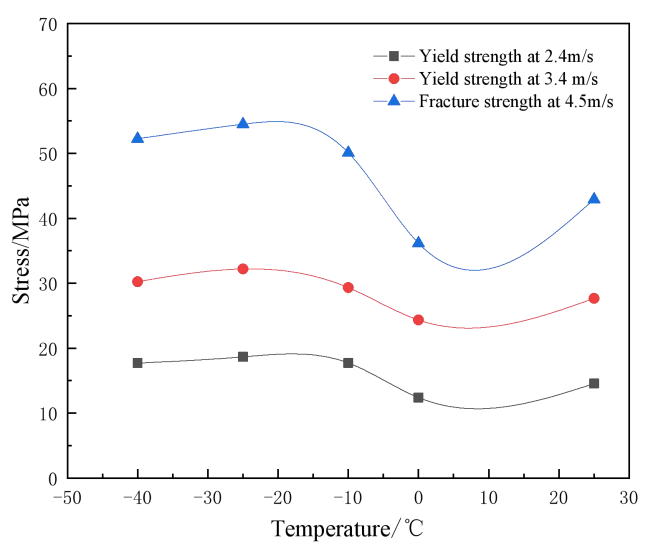

Fig. 11 The yield strength at impact speeds of $2.4 \mathrm{~m} / \mathrm{s}$ and $3.4 \mathrm{~m} / \mathrm{s}$, and the relationship between the fracture strength and temperature at impact speeds of $4.5 \mathrm{~m} / \mathrm{s}$

It can be seen from Fig. 11 that the temperature of piezoelectric ceramics decreases from the temperature of $25^{\circ} \mathrm{C}$ to $0{ }^{\circ} \mathrm{C}$, and the yield and fracture strengths decrease with the decrease of temperature. With the continuous decrease of temperature, the yield and fracture strengths of piezoelectric ceramics increase gradually and reach the stable at the temperature of $-30^{\circ} \mathrm{C}$, and the temperature of $-25^{\circ} \mathrm{C}$ is $26.94 \%$ higher than that at the temperature of $25^{\circ} \mathrm{C}$. According to the fracture morphology of ceramics in Figs. 9 and 10, the increase of steady-state fracture toughness at low temperature may be due to the contribution of phase transformation toughening component [8].

\subsection{Influence of temperature on open circuit discharge characteristics of PZT-5H}

Considering the electric effect of piezoelectric ceramics, the electric performance of 
low-temperature piezoelectric ceramics is tested. The output voltage induced by the temperature change of piezoelectric ceramics is small and the magnitude difference between the output voltage caused by temperature change and the output voltage of piezoelectric effect is $3 \sim 4$ orders, so the electric effect can be ignored.

\subsubsection{Electrical characteristics}

When the piezoelectric ceramics are polarized by an external force along the polarization direction, the charge adsorbed on both ends of the piezoelectric ceramics is released, and after the external force is unloaded, the piezoelectric ceramics return to the uncharged state. The charge density is directly proportional to the applied force, which is called the positive piezoelectric effect. The piezoelectric equation can be expressed as

$$
Q=d_{33} F
$$

Where, $Q$ is the amount of released charge, $d_{33}$ is the piezoelectric strain constant in the polarization direction, and $F$ is the external force along the polarization direction. Piezoelectric ceramics can be equivalent to capacitance, then there is $Q=C U$, where $U$ is the output voltage, $C$ is the capacitance of piezoelectric ceramics. According to the expression of capacitance $C=\varepsilon_{0} \varepsilon_{r} A_{0} / l_{0}$, the relationship can be expressed as

$$
U=\frac{Q l_{0}}{\varepsilon_{0} \varepsilon_{r} A_{0}}
$$

Substituting equation (12) into equation (13), the relationship can be expressed as

$$
U=g_{33} l_{0} \sigma
$$

In formula (14), $g_{33}$ is the voltage piezoelectric constant in the polarization direction.

Figs. 12 and 13 show the time history curves of stress and output voltage at the temperatures of $25^{\circ} \mathrm{C}$ and $-40^{\circ} \mathrm{C}$ with impact velocities of $2.4 \mathrm{~m} / \mathrm{s}$ and $4.5 \mathrm{~m} / \mathrm{s}$, respectively.
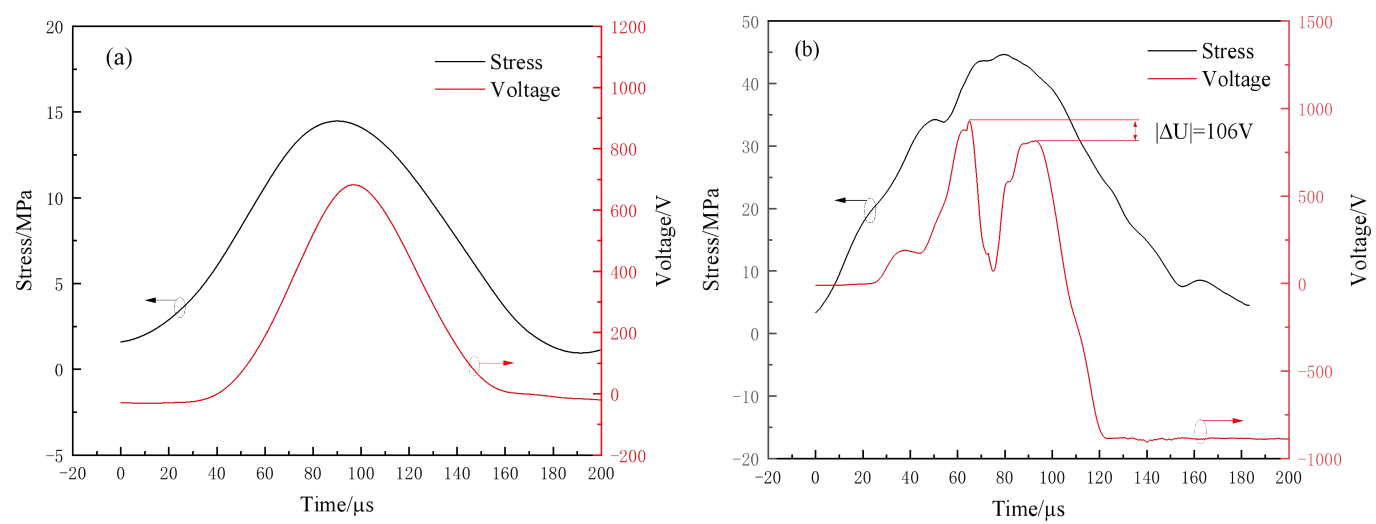

Fig. 12 The time history curves of stress and output voltage at the temperature of $25^{\circ} \mathrm{C}$ (a) The impact velocity $2.4 \mathrm{~m} / \mathrm{s}$, (b) The impact velocity $4.5 \mathrm{~m} / \mathrm{s}$ 

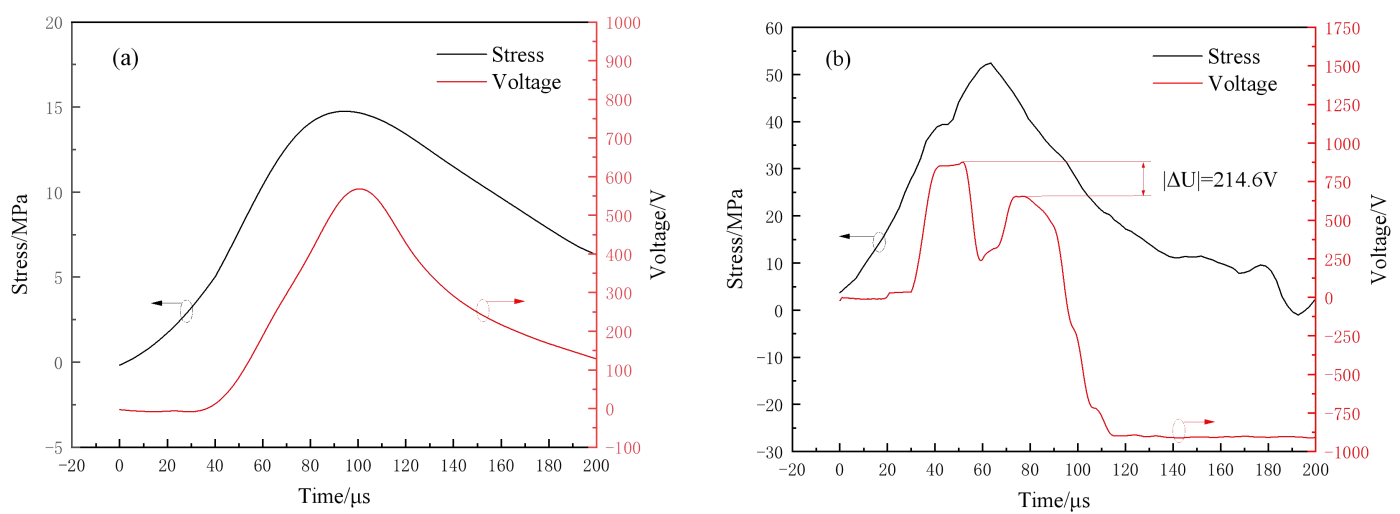

Fig. 13 The time history curves of stress and output voltage at the temperature of $-40^{\circ} \mathrm{C}$ (a) The impact velocity $2.4 \mathrm{~m} / \mathrm{s}$, (b) The impact velocity $4.5 \mathrm{~m} / \mathrm{s}$

From Figs. 12 and 13, it can be seen that the output voltage of both ends of the piezoelectric ceramic is changed with the applied stress in the linear region at the temperatures of -40 and $25^{\circ} \mathrm{C}$. When the piezoelectric ceramic breaks, the voltage drops suddenly, and there are two different peaks in the voltage time history curve, and the second voltage peak is smaller than the first voltage peak, while the difference of the voltage peak before and after the temperature of $-40{ }^{\circ} \mathrm{C}$, fracture is larger than that at the temperature of 25. Piezoelectric ceramic, as a porous material, impurity containing heterogeneous material, its internal micro hole defects and impurity defects will affect the macroscopic discharge, and the temperature will change the internal structure of piezoelectric ceramics, thereby increasing the peak voltage difference. Fig. 14 shows the local SEM iconographies and the energy spectrums of the piezoelectric ceramics, which Fig. 14(a) shows the blowhole defects on the surface of the piezoelectric ceramics (the red circle in Fig. 14(a)).

(a)
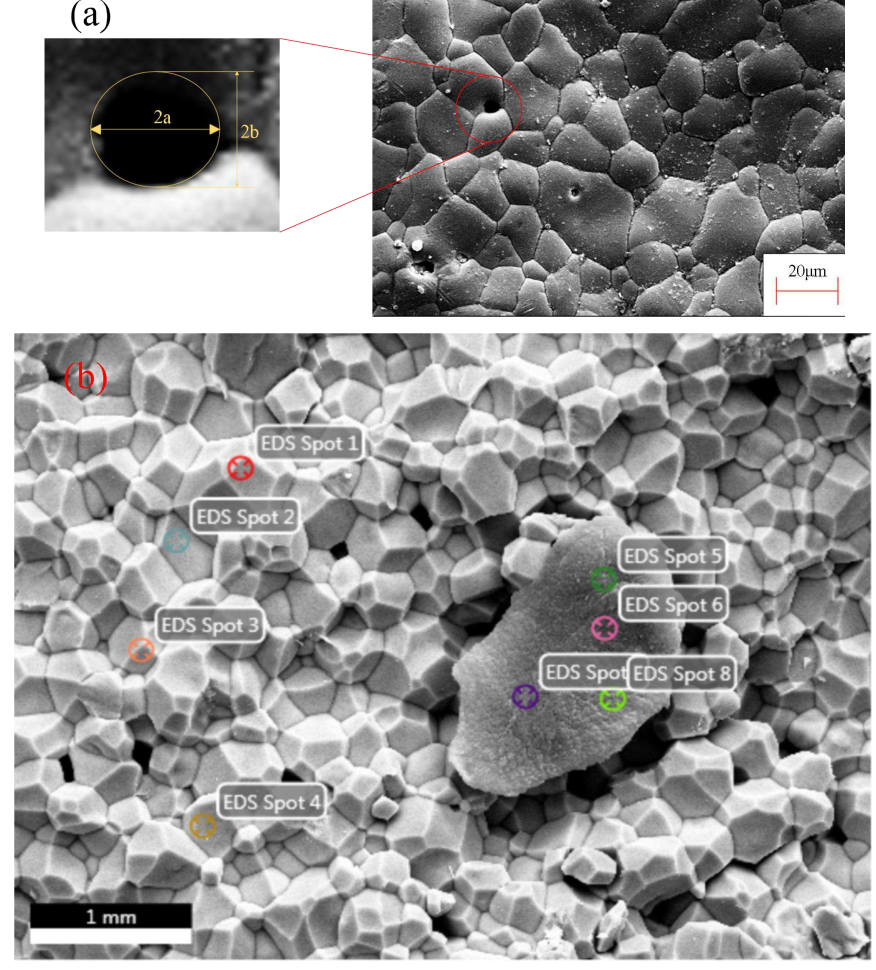

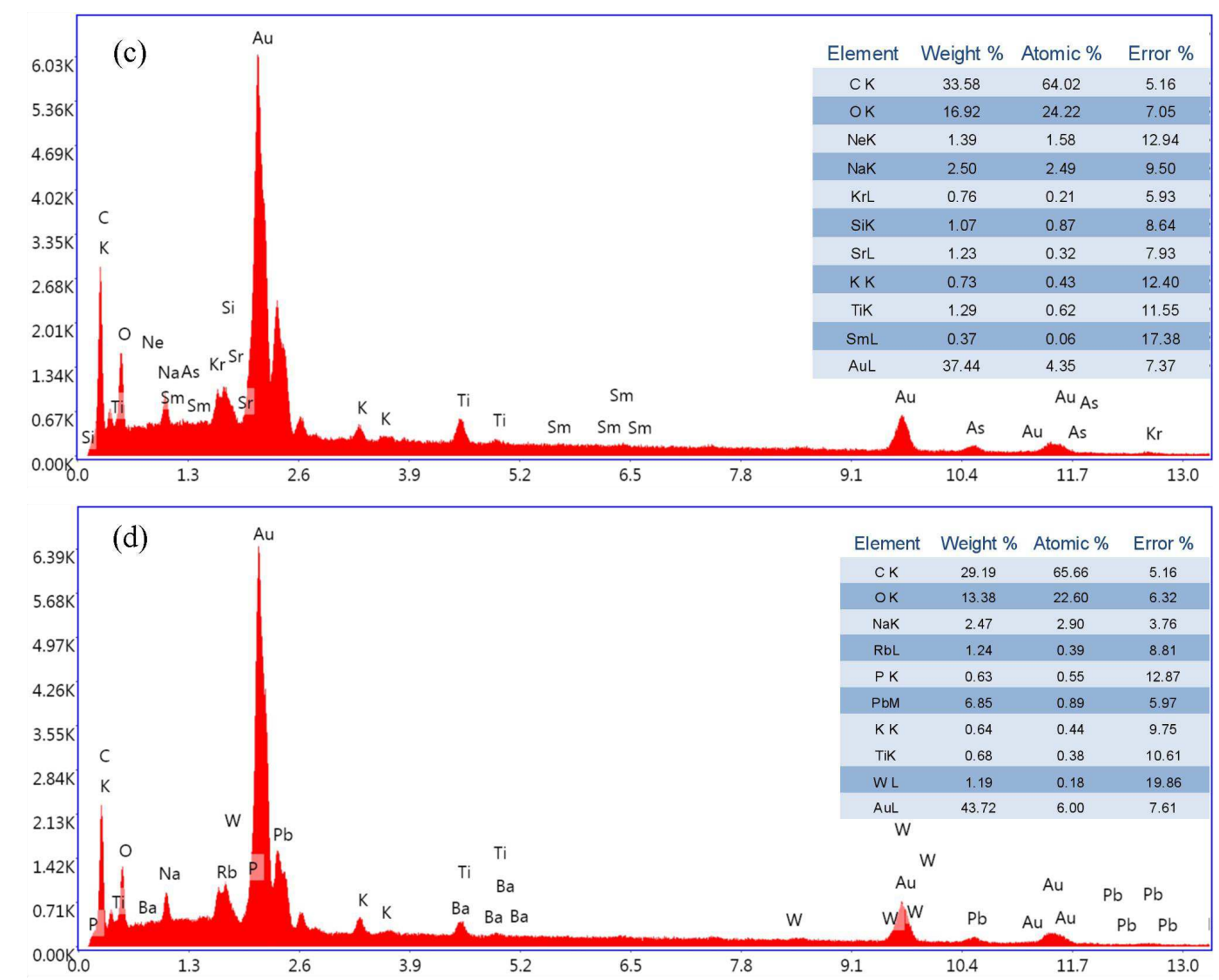

Fig. 14 (a) The local SEM iconography of the piezoelectric ceramics $(500 \times)$, (b) The local SEM iconography of the piezoelectric ceramics $(5 \times)$, (c) Energy spectrum of EDS spot 7 of the local SEM iconography of the piezoelectric ceramics $(5 \times)$,(d) Energy spectrum of EDS spot 8 the local SEM iconography of the piezoelectric ceramics $(5 \times)$

In fact, the dielectric constant of the air or ionized gas inside the piezoelectric ceramics is limited, but it can withstand higher electric field strength, so it has a certain conductivity. Emceeing $^{[27]}$ and Dunn ${ }^{[28]}$ used the dielectric ellipse model to describe the defects. The long and short axises of the ellipse are $2 \mathrm{a}$ and $2 \mathrm{~b}$, respectively. The dielectric constants of the ellipse and the matrix were $\varepsilon_{f}$ and $\varepsilon_{m}$, respectively. The conductivity of the defects is described by the electric field permeability parameter $\kappa$

$$
\kappa=\frac{a \varepsilon_{f}}{b \varepsilon_{m}}
$$

The porosity defects shown in Fig. 14 (a) can be regarded as ellipses along the polarization direction. The dielectric constants of the interior and the substrate of the ellipse are the dielectric constants of the air at the temperature of $25^{\circ} \mathrm{C}$, respectively, and the long and short axises of the ellipse are measured according to the scale to obtain $\kappa=3.54 \times 10^{-4}$. In this paper, the conductivity of piezoelectric ceramics at the temperature of $25^{\circ} \mathrm{C}$ is measured to be $\sigma_{e}=96.4 \times 10^{-4} \Omega^{-1} \cdot \mathrm{m}^{-1}$. Comparing the values of $\kappa$ and $\sigma_{e}$, it can be found that pore defects and crack gaps will affect the voltage output of piezoelectric ceramics, and the influence degree is related to pore size, pore number and crack gap size. The reduction of temperature makes the grains inside the piezoelectric ceramics shrink, and the size of the pores increases, which increases the output voltage difference. 
Fig. 14 (b) shows the local SEM iconography of the piezoelectric ceramics with EDS spots $1 \sim 8$ are the energy spectrum analysis spots, and Fig. 14 (c) and (d) are the energy spectrum of EDS spot 7 and EDS spot 8, respectively. In the practical operation of energy spectrum analysis, because the ceramic is a poor conductor, an additional conductive thin layer material needs to be sputtered on the inner surface of the measured ceramic (the material used in this paper is gold), so the proportion of Au element in Fig. 14 (c) and (d) are the most. The energy spectrum analysis shows that there are $\mathrm{C}, \mathrm{K}, \mathrm{Na}, \mathrm{Ne}, \mathrm{Kr}, \mathrm{Si}$ and other elements in the piezoelectric ceramics, which indicates that there are impurities in the piezoelectric ceramics.

There is also a discharge phenomenon of piezoelectric ceramics excited by impurities. The electric field formed by the impact discharge makes the charge temperature excited by ceramic impurities reach a critical level and discharge, and the decrease of temperature further affects the voltage output of piezoelectric ceramics.

\subsubsection{The freezing effect}

At room temperature, when the piezoelectric ceramics are polarized by an external force along the polarization direction, the charge absorbed by both ends of the electrode is released, and the voltage returns to zero potential as the external force unloading. Due to the ferroelectric of piezoelectric ceramics, when the piezoelectric ceramics break, the voltage will increase reversely to the opposite number of voltage peak value caused by external force. Figs. 15 and 16 show that time history curves of output voltage with impact velocity of $3.4 \mathrm{~m} / \mathrm{s}$ and $4.5 \mathrm{~m} / \mathrm{s}$ at the temperatures of $-25{ }^{\circ} \mathrm{C}$ and 25 , respectively, only the piezoelectric ceramic is damaged at the temperature of $25^{\circ} \mathrm{C}$, and the voltage at both ends of piezoelectric ceramic will not return to zero potential after the piezoelectric ceramic is discharged. However, in the case of $-25^{\circ} \mathrm{C}$, there is a phenomenon that the voltage at both ends of the piezoelectric ceramic does not return to zero potential and increases reversely after discharge, including the impact at the temperature of $0{ }^{\circ} \mathrm{C}$ and the impact velocities of $3.4 \mathrm{~m} / \mathrm{s}$ and $4.5 \mathrm{~m} / \mathrm{s}$ (that is, the piezoelectric ceramic does not break after impact), respectively. This is related to the rotation of domain in piezoelectric ceramics. In this paper, the phenomenon that the output voltage of the unbroken piezoelectric ceramics does not return to zero potential and increases reversely to the opposite number of voltage peaks after unloading under low temperature is called the freezing effect.

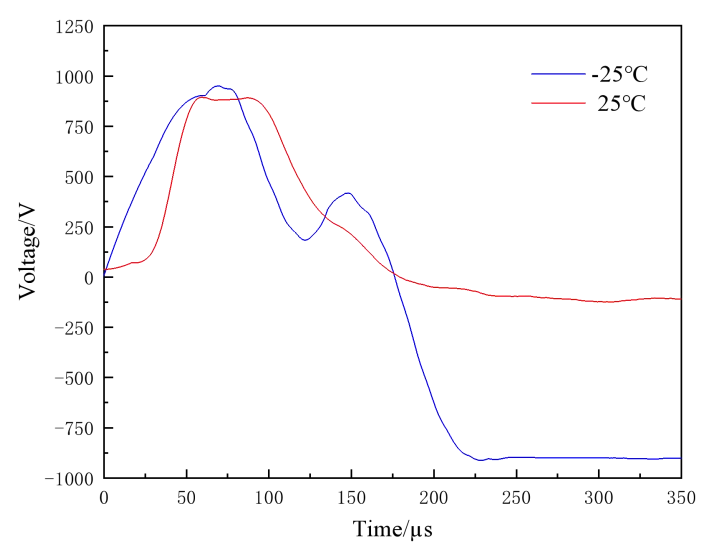

Fig. 15 The voltage time history curve at impact speed of $3.4 \mathrm{~m} / \mathrm{s}$ at $-25^{\circ} \mathrm{C}$ and $25^{\circ} \mathrm{C}$

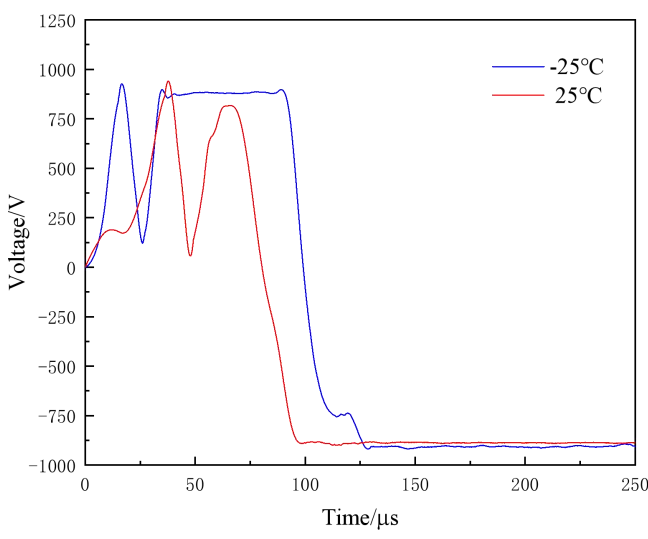

Fig. 16 The voltage time history curve at impact speed of $4.5 \mathrm{~m} / \mathrm{s}$ at $-25^{\circ} \mathrm{C}$ and $25^{\circ} \mathrm{C}$

According to the space charge theory of Kazantzakis ${ }^{[29]}$, the space charge in the lattice is mostly vacancy and atom, and the space charge generated by polarization generally exists near the grain boundary or domain wall, as shown in Fig. 17. 

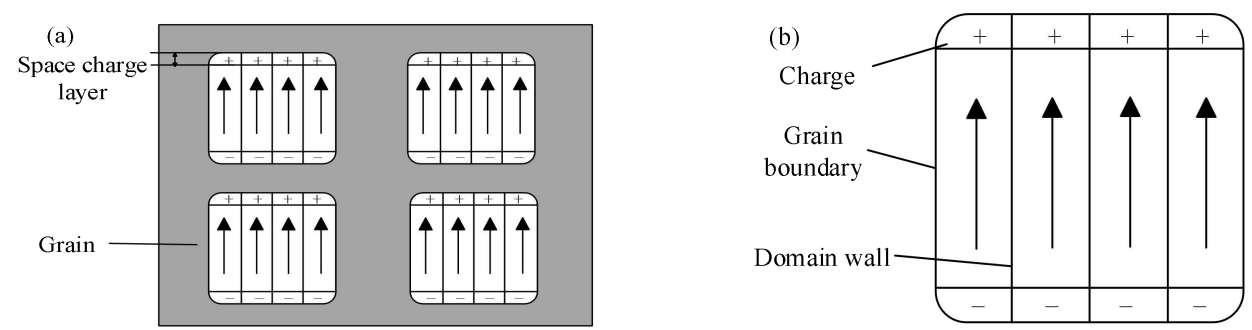

Fig. 17 (a) The porous polarized grain structure, (b) The crystal domain structure of piezoelectric ceramics after polarization

The discharge of piezoelectric ceramics is composed of the movement of charge in the lattice, and the movement of charge is realized through the movement of vacancies and interstitial atoms in the lattice. The movement of atoms jumps from one lattice position to another, while the jumping of vacancy and interstitial atoms depends on thermal fluctuation ${ }^{[22]}$, so it is closely related to temperature. Taking the gap atom as an example, the gap atom is in a lattice position with a minimum relative potential energy, and there is a maximum potential energy, i.e. potential barrier, between the gap atom and the adjacent gap. The gap atom generally vibrates in the vicinity of the minimum potential energy. The gap atom must cross the barrier to jump the adjacent gap. According to the statistics of the thermal fluctuation probability, the jump rate of the gap atom shows that the movement of the gap atom increases exponentially with the increase of temperature ${ }^{[22]}$. According to the similar analysis, the vacancy motion can also get similar results.

It can be seen from Fig. 17 that the electric field generated by the impact pressure of piezoelectric ceramics has the opposite effect on the space charge layer in the grain and the domain, thus hindering the domain rotation, the grains of piezoelectric ceramics shrink at low temperature, the space charge layer increases, and the domain wall cannot keep up with the electric field change caused by the impact pressure, which makes it more difficult to rotate. Therefore, at low temperature, the impact pressure of piezoelectric ceramics produces freezing effect without fracture.

The effect of grain size on piezoelectric properties is greater than that on dielectric properties. PZT-5H piezoelectric properties are better at room temperature than at low temperature, which is consistent with the experimental results in literature [5].

\subsubsection{Electrical output characteristics}

When piezoelectric ceramics work in linear region, due to electric field strength $E=U / l_{0}$, the relationship can be expressed as

$$
E=g_{33} \sigma
$$

Fig. 18 shows the stress time history curve at the impact velocity of $3.4 \mathrm{~m} / \mathrm{s}$ and the temperature of $0{ }^{\circ} \mathrm{C}$, and the electric field strength time history curve at the impact velocity of $3.4 \mathrm{~m} / \mathrm{s}$ and different temperatures. In the linear region of the figure, the relationship between the piezoelectric voltage constant $g_{33}$ and the temperature at different impact speeds are derived from equation (16), as shown in Fig. 19, At the same impact velocity, the piezoelectric voltage constant increases with the decrease of temperature. At the temperature of $25{ }^{\circ} \mathrm{C}$, there is no significant difference in the piezoelectric voltage constant at different impact velocities. At low temperature, the impact velocity has significant influence on the piezoelectric voltage constant. 


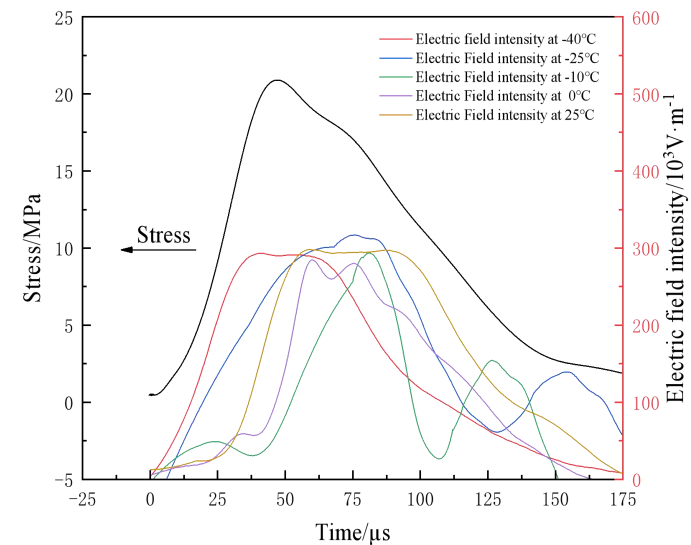

Fig. 18 The time history curves of stress and electric field strength at impact velocity of $3.4 \mathrm{~m} / \mathrm{s}$ at $0{ }^{\circ} \mathrm{C}$, and at different temperatures

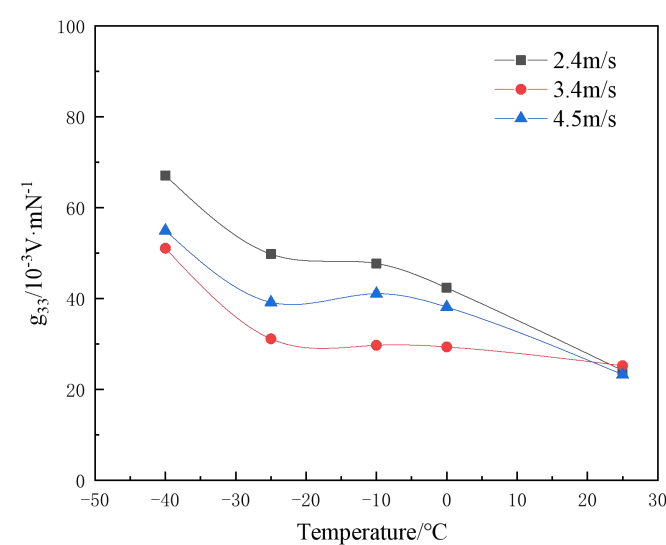

Fig. 19 The relationship between piezoelectric voltage constant $g_{33}$ and temperature at different impact velocities

It can be seen from Fig. 19 that when the impact speed is constant, the piezoelectric voltage constant $g_{33}$ decreases with the increase of temperature, and the impact stress increases with the increase of impact velocity; when the temperature is fixed, the piezoelectric voltage constant $g_{33}$ changes with the impact velocity, but the impact velocity has no obvious influence on the piezoelectric voltage constant at room temperature.

The dynamic piezoelectric constant of piezoelectric ceramics in the linear working area is $g_{33}=U / l_{0} \sigma$ by means of equation (14). Among them, the static piezoelectric constant $g_{33}^{\prime}=d_{33} / \varepsilon_{0} \varepsilon_{r}$ and the dynamic piezoelectric constant $g_{33}=d_{33} / \varepsilon_{0} \varepsilon_{r}$. The ratio of dynamic piezoelectric constant $g_{33}$ to static piezoelectric constant $g_{33}^{\prime}$ is $\eta=g_{33} / g_{33}^{\prime}=1$. Therefore, the relative permittivity $\varepsilon_{r}$ of piezoelectric ceramics should be kept constant in the impact process. The piezoelectric constants of different temperatures at impact speeds of $2.4 \mathrm{~m} / \mathrm{s}, 3.4 \mathrm{~m} / \mathrm{s}$ and $4.5 \mathrm{~m} / \mathrm{s}$ are shown in tables $3 \sim 5$.

Table 3 The piezoelectric constant at impact velocity of $2.4 \mathrm{~m} / \mathrm{s}$

\begin{tabular}{|c|c|c|c|c|c|}
\hline$T /{ }^{\circ} \mathrm{C}$ & $\varepsilon_{r}^{\prime}$ & $d_{33} / 10^{-12} \mathrm{C} \cdot \mathrm{N}^{-1}$ & $g_{33} / 10^{-3} \mathrm{~V} \cdot \mathrm{mN}^{-1}$ & $g_{33}^{\prime} / 10^{-3} \mathrm{~V} \cdot \mathrm{mN}^{-1}$ & $\eta$ \\
\hline-40 & 2306.12427 & 821.9384019 & 67.05737821 & 40.27293624 & 1.665072986 \\
\hline-25 & 2770.08583 & 732.8443672 & 49.77113415 & 29.89339617 & 1.664954155 \\
\hline-10 & 2980.64057 & 755.6639655 & 47.69459831 & 28.64678139 & 1.664919966 \\
\hline 0 & 3096.88549 & 696.6366427 & 42.32638988 & 25.41779639 & 1.665226569 \\
\hline 25 & 3199.27261 & 411.534613 & 24.15177768 & 14.53489336 & 1.661641203 \\
\hline \multicolumn{6}{|c|}{ Table 4 The piezoelectric constant at impact velocity of $3.4 \mathrm{~m} / \mathrm{s}$} \\
\hline$T /{ }^{\circ} \mathrm{C}$ & $\varepsilon_{r}^{\prime}$ & $d_{33} / 10^{-12} \mathrm{C} \cdot \mathrm{N}^{-1}$ & $g_{33} / 10^{-3} \mathrm{~V} \cdot \mathrm{mN}^{-1}$ & $g_{33}^{\prime} / 10^{-3} \mathrm{~V} \cdot \mathrm{mN}^{-1}$ & $\eta$ \\
\hline-40 & 2306.12427 & 625.6501192 & 51.04331 & 30.65529887 & 1.665072985 \\
\hline-25 & 2770.08583 & 458.1229191 & 31.11342 & 18.68725548 & 1.66495396 \\
\hline-10 & 2980.64057 & 470.8404227 & 29.71763 & 17.84928655 & 1.664919767 \\
\hline 0 & 3096.88549 & 483.1466269 & 29.35512 & 17.6283041 & 1.665226549 \\
\hline 25 & 3199.27261 & 429.4237078 & 25.20164 & 15.16671406 & 1.661641402 \\
\hline
\end{tabular}

Table 5 The piezoelectric constant at impact velocity of $4.5 \mathrm{~m} / \mathrm{s}$

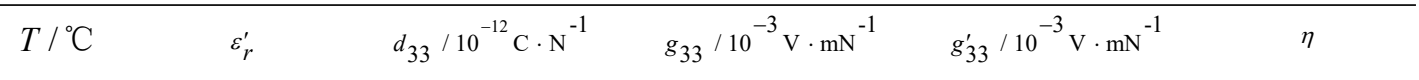




\begin{tabular}{cccccc}
\hline-40 & 2306.12427 & 673.6303478 & 54.95774976 & 33.00621067 & 1.665072986 \\
-25 & 2770.08583 & 576.6116138 & 39.16058479 & 23.52051837 & 1.664954155 \\
-10 & 2980.64057 & 650.5670289 & 41.06128455 & 24.66261766 & 1.664919966 \\
0 & 3096.88549 & 627.5364981 & 38.12798933 & 22.89657758 & 1.665226569 \\
25 & 3199.27261 & 396.4192695 & 23.26470183 & 14.00103813 & 1.661641203 \\
\hline
\end{tabular}

It can be seen from tables $3 \sim 5$ that the ratio of dynamic piezoelectric constant $g_{33}$ to static piezoelectric constant $g_{33}^{\prime}$ is $\eta=g_{33} / g_{33}^{\prime} \neq 1 \approx 1.66$, which indicates that the relative permittivity of piezoelectric ceramics varies with temperature is independent of temperature.

\section{Conclusion}

In this paper, the static electrical parameters of PZT-5H were measured at the temperature of $-40 \sim 25^{\circ} \mathrm{C}$, and the mechanical test system of liquid nitrogen cooling/Hopkinson piezoelectric bar and the voltage output test system of piezoelectric ceramics were established. The stress load and electrical output characteristics of piezoelectric ceramics were measured at different temperatures and impact speeds. The following conclusions can be drawn:

(1) With the temperature of PZT-5H decreasing from the temperature of $25^{\circ} \mathrm{C}$ to $-40{ }^{\circ} \mathrm{C}$, the resistivity and relative permittivity decreased, and the relative permittivity decreases significantly. But the relaxation time increases with the temperature decreasing.

(2) Different from the battlement of some metals and polymers at low temperature, the mechanical properties of PZT-5H can still maintain a certain level or even have a significant improvement trend at low temperature.

(3) When the piezoelectric ceramic breaks, the pore defects and cracks at the fracture site are one of the reasons for the difference of the two output voltage peak values. Reducing the temperature makes the crystal size of the piezoelectric ceramic narrow, and the pore size increases the difference with the increase of the voltage peak values.

(4) At low temperature, the freezing effect of PZT-5H is microcosmic because the grain size reduction at low temperature affects the charge movement in the grain and thus hinders the domain rotation. At the macro level, the grain size has a greater influence on the piezoelectric properties than that of the dielectric properties. The piezoelectric properties of PZT-5H at room temperature are better than those at low temperature;

(5) When the impact velocity is fixed, the piezoelectric voltage constant increases with the decrease of temperature, and when the temperature is fixed, the piezoelectric voltage constant decreases with the increase of impact velocity. At room temperature, the impact velocity has no obvious influence on the piezoelectric voltage constant. In the elastic region of PZT-5H, the relative dielectric constant of piezoelectric ceramics changes significantly compared with that of static state. The dynamic piezoelectric constant is about 1.66 times that of the static piezoelectric constant.

The authors declare that we have no conflict of interest.

The data that support the findings of this study are available from the corresponding author upon reasonable request.

\section{Acknowledgements}

This research was supported by the National Natural Science Foundation of China (grant No. 11472178). Thanks to the reaserch group in Intense Dynamic Loading Research Center of Shenyang Ligong University for their support during the experiments, especially for the efforts of graduate students in experimental measurements. 


\section{References}

[1] Jaffe B, Roth R S, Marzullo S. Piezoelectric Properties of Lead Zirconate-Lead Titanate Solid-Solution Ceramics. Journal of Applied Physics, 1954, 25(6):809-0.

[2] John E McKinney, C Stuart Bowyer. Determination of Piezoelectric Properties as a Function of Pressure and Temperature. Journal of the Acoustical Society of America, 1960, 32(1).

[3] Qiming Zhang, Haimin Wang, N Kim, et al. Direct evaluation of domain-wall and intrinsic contributions to the dielectric and piezoelectric response and their temperature dependence on lead zirconate-titanate ceramics. Journal of Applied Physics, 1994, 75(1):454-0.

[4] Beatriz Noheda, Noé Cereceda, Tomás Iglesias, et al. Composition dependence of the ferroelectricparaelectric transition in the mixed system PbZr1-XTiXO ${ }_{3}$. Physical Review B, 1995, 51(22):16388.

[5] Hooker M W. Properties of PZT-Based Piezoelectric Ceramics Between -150 and $250{ }^{\circ} \mathrm{C}$. NASA/CR-1998-208708 1998.

[6] Panda P K, Kannan T S, Dubois J, et al. Thermal shock and thermal fatigue study of ceramic materials on a newly developed ascending thermal shock test equipment. Science and Technology of Advanced Materials, 2002, 3(4):327-334.

[7] Andryushina, I. N, Reznichenko, et al. The PZT system (PbTixZr1 $\left.1_{-\mathrm{x}} \mathrm{O}_{3}, 0 \leqslant \mathrm{x} \leqslant 1.0\right)$ : Dielectric response of; solid solutions in broad temperature $(10 \leqslant \mathrm{~T} \leqslant 1000 \mathrm{~K})$ and frequency; $\left(10^{(-2)} \leqslant \mathrm{f} \leqslant 10^{(7)} \mathrm{Hz}\right)$ ranges (Part 4). Ceramics International, 2013, 39(4):3979-3986.

[8] Xue Weijiang. Study on phase transformation, fracture mechanism and properties of structural ceramics at low temperature . Tsinghua University, 2013.

[9] Lan Chunfeng, Nie Hengchang, Chen Xuefeng, et al. Low temperature phase structure and electrical properties of dense PZT 95/5 Ferroelectric Ceramics. Inorganic, 2013, 28 (5).

[10] Anand S , Arockiarajan A . Temperature dependent ferroelectric and ferroelastic behaviour of PZT wafers. Ceramics International, 2016:S0272884216310513.

[11] Wanqiang Cao, Peichao Liu, YongChen, Ruikun Pan, Yajun Qi. Influence of domain lag on residual polarization in ferroelectrics . Acta physica Sinica, 2016, 65 (13): 262-268.

[12] Belovickis J , Ivanov M, Samulionis V, et al. Dielectric, Ferroelectric, and Piezoelectric Investigation of Polymer-Based P(VDF-TrFE) Composites[J]. Physica Status Solidi, 2018, 255(3):1700196.

[13] Enling Tang, Yue Li, Ruizhi Wang, et al. Experimental Research on Piezoelectric Ceramics Activating Micro-sized Thermochemical Battery. International Journal of Applied Ceramic Technology, 2019:1-8

[14] Ruizhi Wang, Enling Tang, Guolai Yang. Dynamic Piezoelectric Properties of PZT-5H Under Shock Compression. Physical Status Solidi : Applications and Materials Science, 2019, 216(6): 1800859.

[15] Ruizhi Wang, Enling Tang, Guolai Yang, et al. Experimental Research of Electrical Output Characteristics of Stacked PZT-5H Under High-Overload Conditions. Journal of Electronic Materials, 2019, 48(5): 2737-2744.

[16] Ruizhi Wang, Enling Tang, Guolai Yang, et al. Research on layer-counting experimental simulation system for projectile penetrating multi-layered targets. Measurement, 2020, 151(2): 107108.

[17] Ruizhi Wang, Enling Tang, Guolai Yang, et al. Experimental research on dynamic response of PZT-5H under impact load[J]. Ceramics International(In press) . https://doi.org/10.1016/j. ceramint.2019.09.280.

[18] Flannigan C,Tan C D,Scott J F. Electrical studies of Barkhausen switching noise in ferroelectric lead zirconate titanate (PZT) and $\mathrm{BaTiO}_{3}$ : critical exponents and temperature-dependence.[J]. Journal of physics. Condensed matter: an Institute of Physics journal,2019,32(5).

[19] Wang D , Fotinich Y, Carman G P . Influence of temperature on the electromechanical and fatigue behavior of piezoelectric ceramics[J]. Journal of Applied Physics, 1998, 83(10):1.

[20] Yunfang Lu, et al. Hopkinson bar experiment technology . Beijing: Science Press, 2013. 
[21] Jinxin Fang, Zhiwen Yin. Dielectric physics . Beijing: Science Press, 2000.

[22] Ruqi Han , Kun Huang. Solid state physics . Beijing: Higher Education Press, 1985.

[23] Jingde Li , et al. Dielectric theory . Beijing: Science Press, 2003.

[24] Tao Zeng, Gen-Shui Wang, Xian-Lin Dong, et al.Investigation on FR(LT)-FR(HT) phase transition and pyroelectric properties of porous Zr-rich lead zirconate titante ceramics. Mater. Sci. \& Eng. B, 2007, 140: 5-9.

[25] Anderson P W. Paper given at the All-Union Conference on Dielectrics, Moscow, 1958.

[26] Mitsui T, Tatsuzaki I, Nakamura E, et al. An introduction to the physics of ferroelectrics. Gordon and Breach Science, 1976.

[27] Mcmeeking R M. Electrostrictive stresses near crack-like flaws. Zeitschrift fur Angewandte Mathematik und Physik(ZAMP), 1989, 40(5):615-627.

[28] Dunn M L.The effects of crack face boundary conditions on the fracture mechanics of piezoelectric solids. Engineering Fracture Mechanics, 1994, 48(1):25-39.

[29] Okazaki K, Nagata K. Effects of Grain Size and Porosity on Electrical and Optical Properties of PLZT Ceramics. Journal of the American Ceramic Society, 1973, 56(2):82-86. 


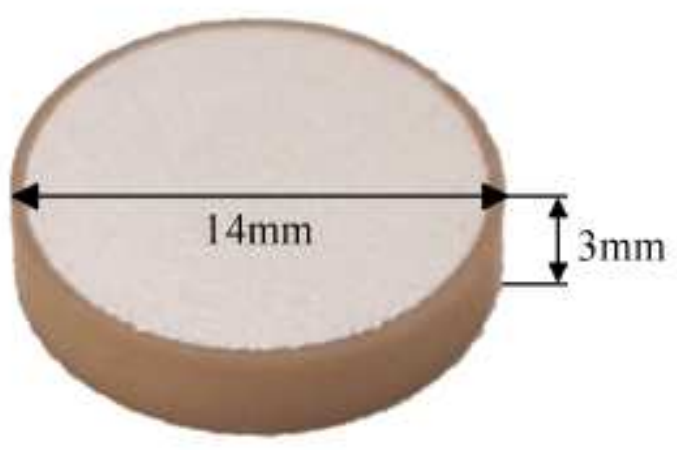

\section{Figure 1}

The physical diagram of PZT-5H

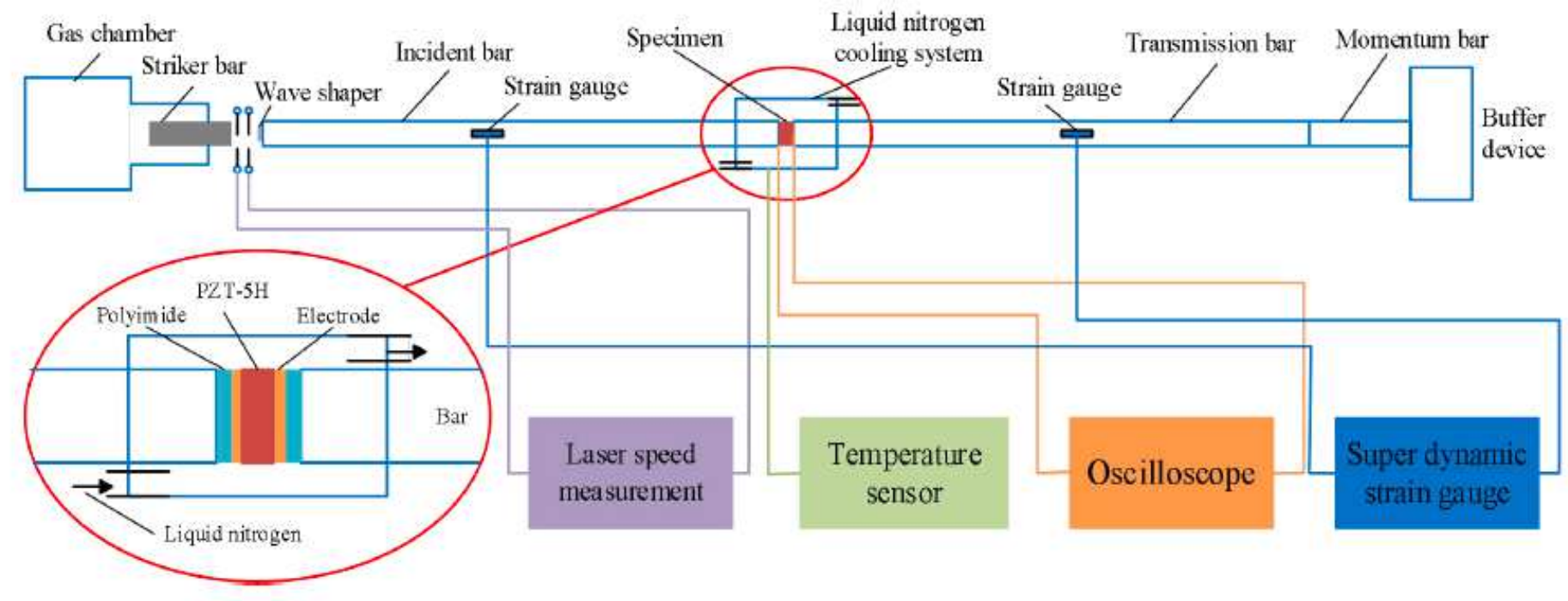

Figure 2

The schematic diagram of the experimental system 


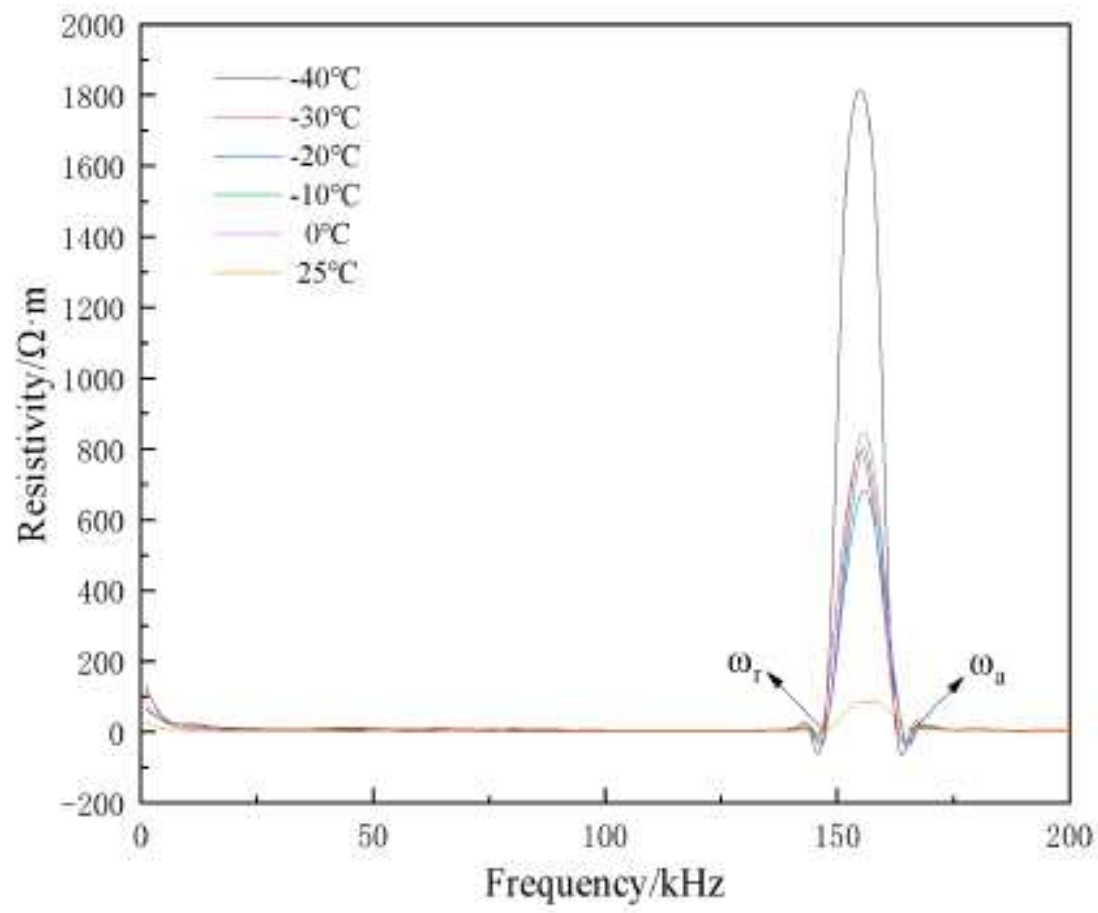

\section{Figure 3}

The resistivity spectrum of PZT-5H at different temperatures

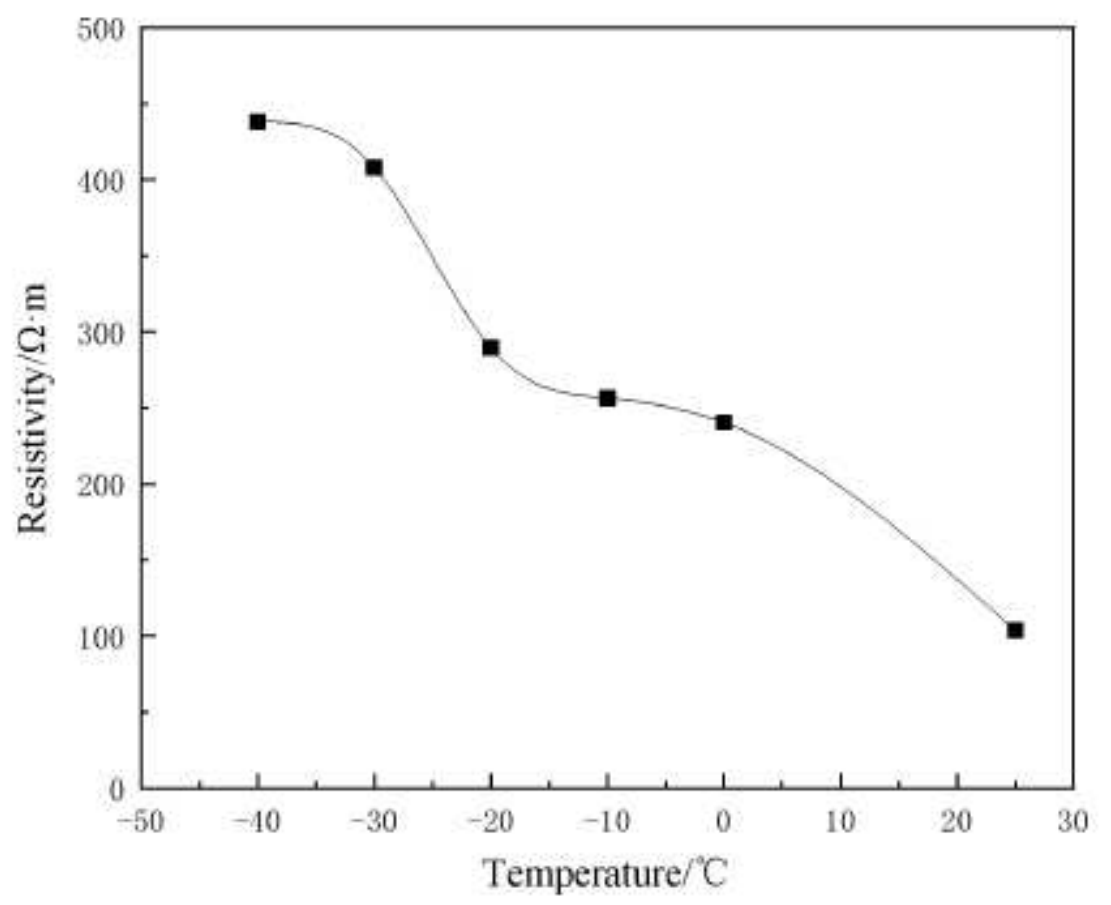

Figure 4

The variation of resistivity with temperature under loading frequency $\omega 0$ 


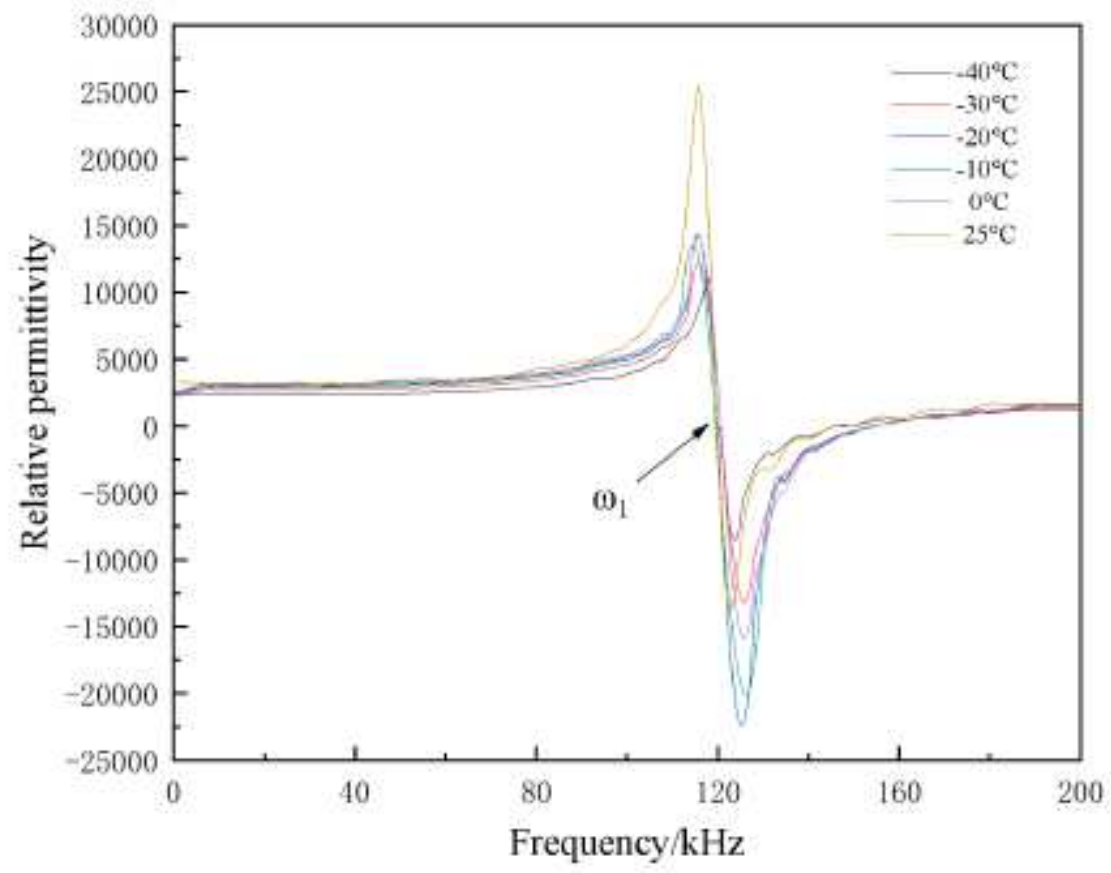

Figure 5

The relative permittivity spectrum of PZT-5H at different temperatures

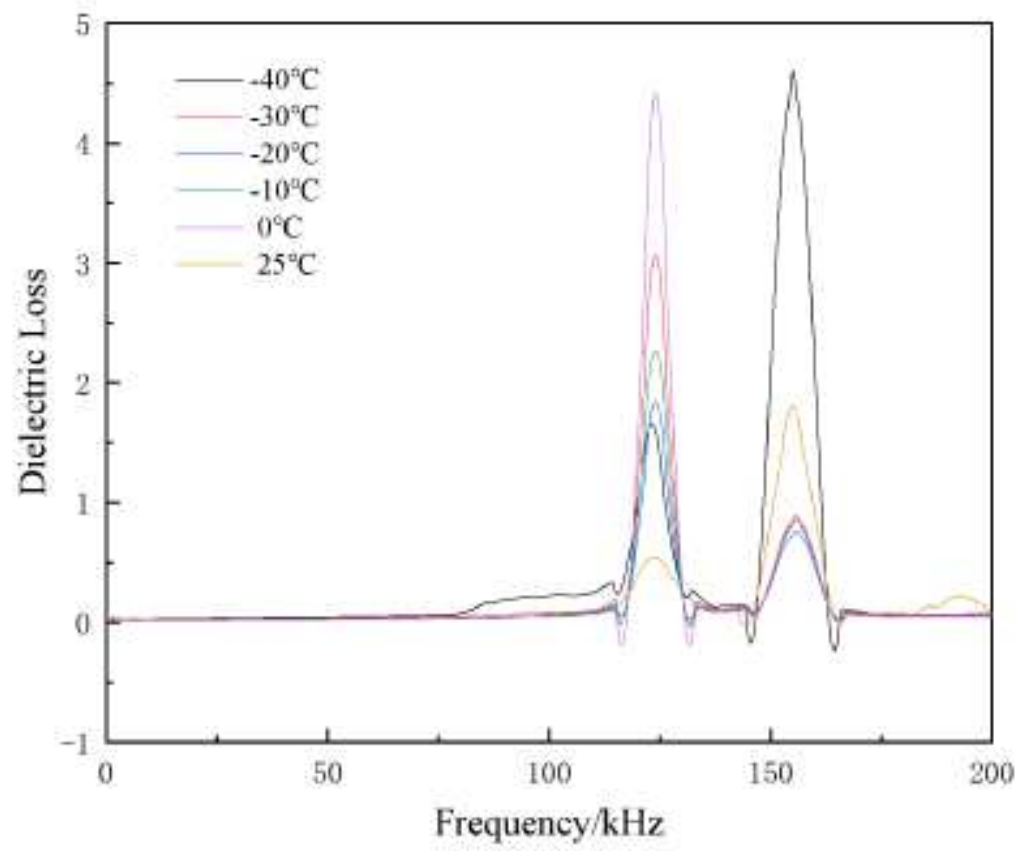

Figure 6

The dielectric loss spectrum of PZT-5H at different temperatures 


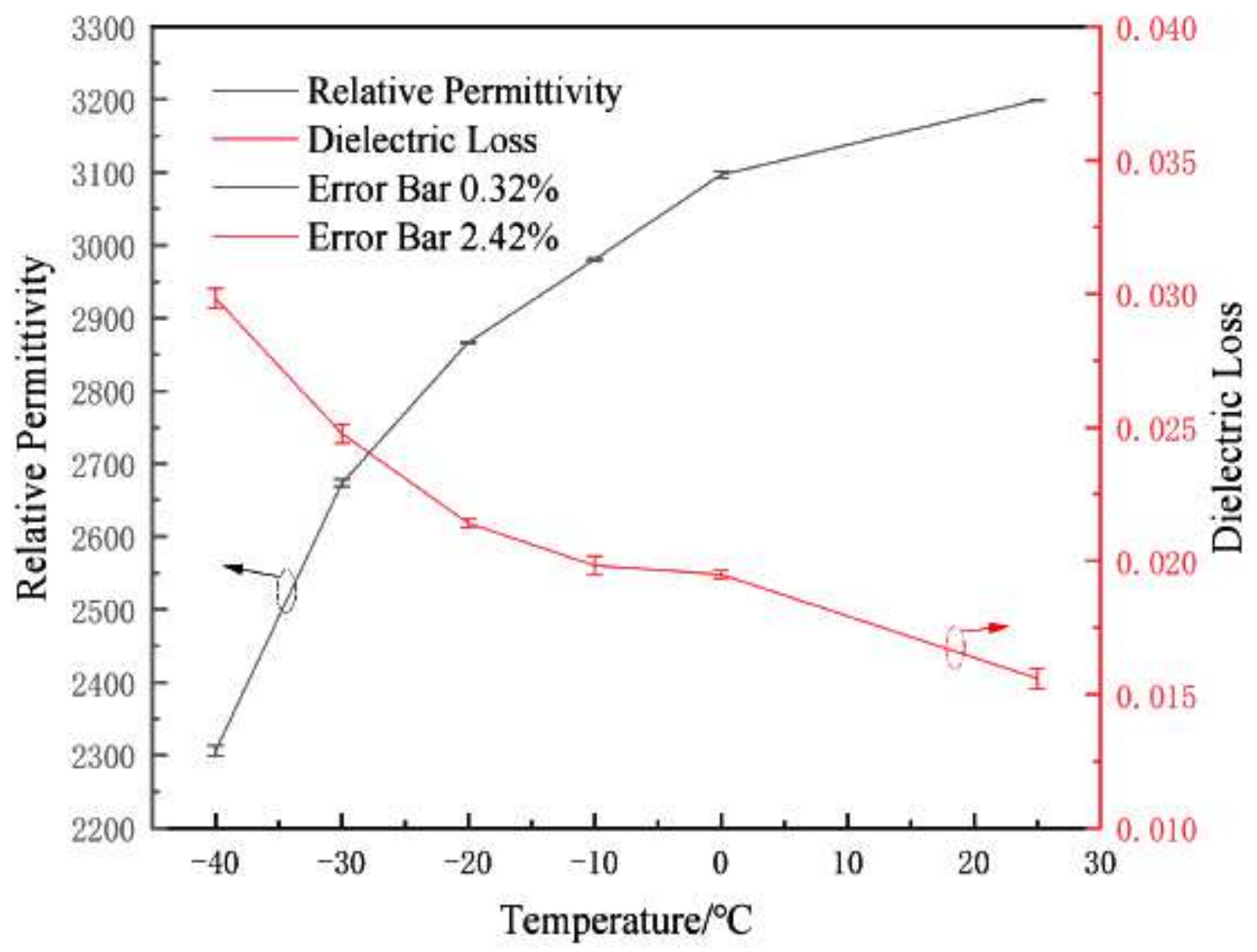

Figure 7

The curves of relative permittivity $\varepsilon^{\prime}$ r, dielectric loss and temperature at loading frequency $\omega 0$ 


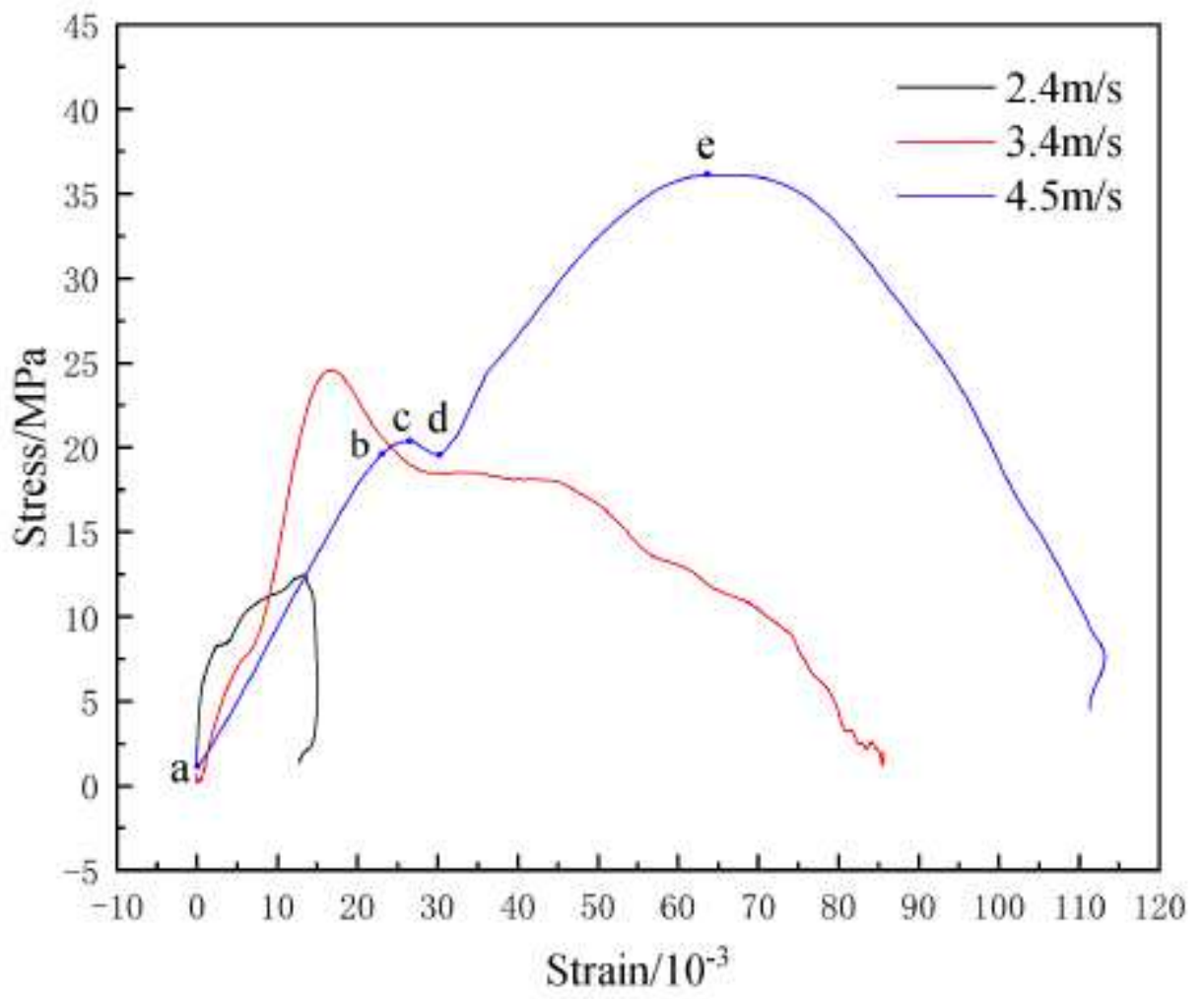

Figure 8

The stress and strain curve of PZT-5H at different impact speeds at the temperature of $0 \mathrm{D}$

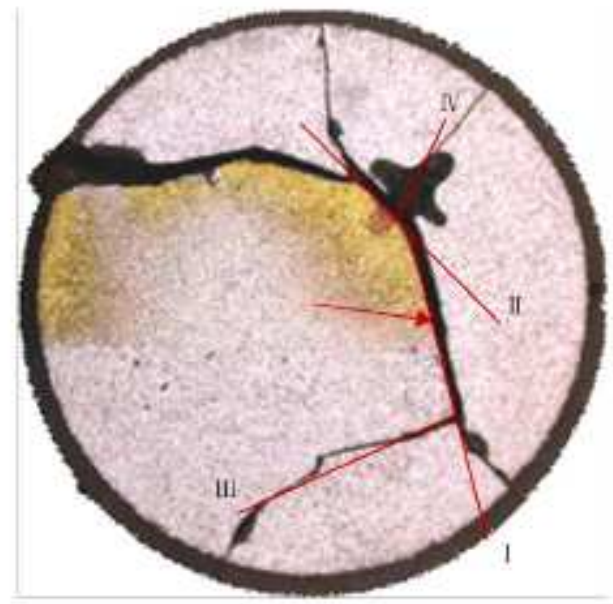

Figure 9

The fracture of piezoelectric ceramics 

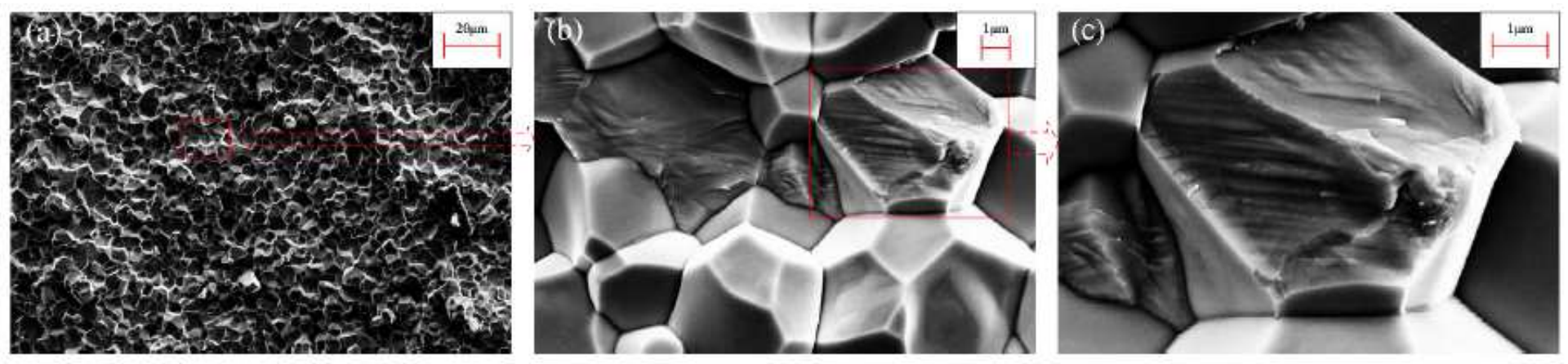

Figure 10

Local SEM of fracture surface of piezoelectric ceramics (a) 500x, (b) 5000x, (c) 5000x

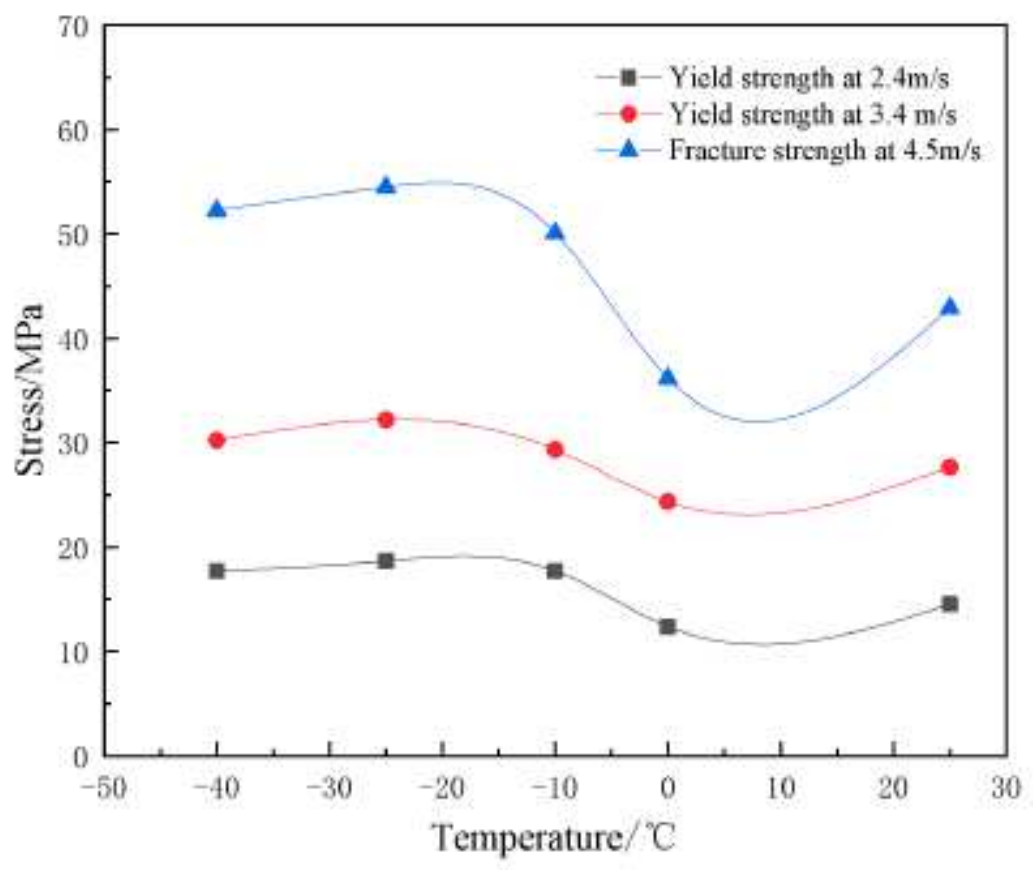

Figure 11

The yield strength at impact speeds of $2.4 \mathrm{~m} / \mathrm{s}$ and $3.4 \mathrm{~m} / \mathrm{s}$, and the relationship between the fracture strength and temperature at impact speeds of $4.5 \mathrm{~m} / \mathrm{s}$ 

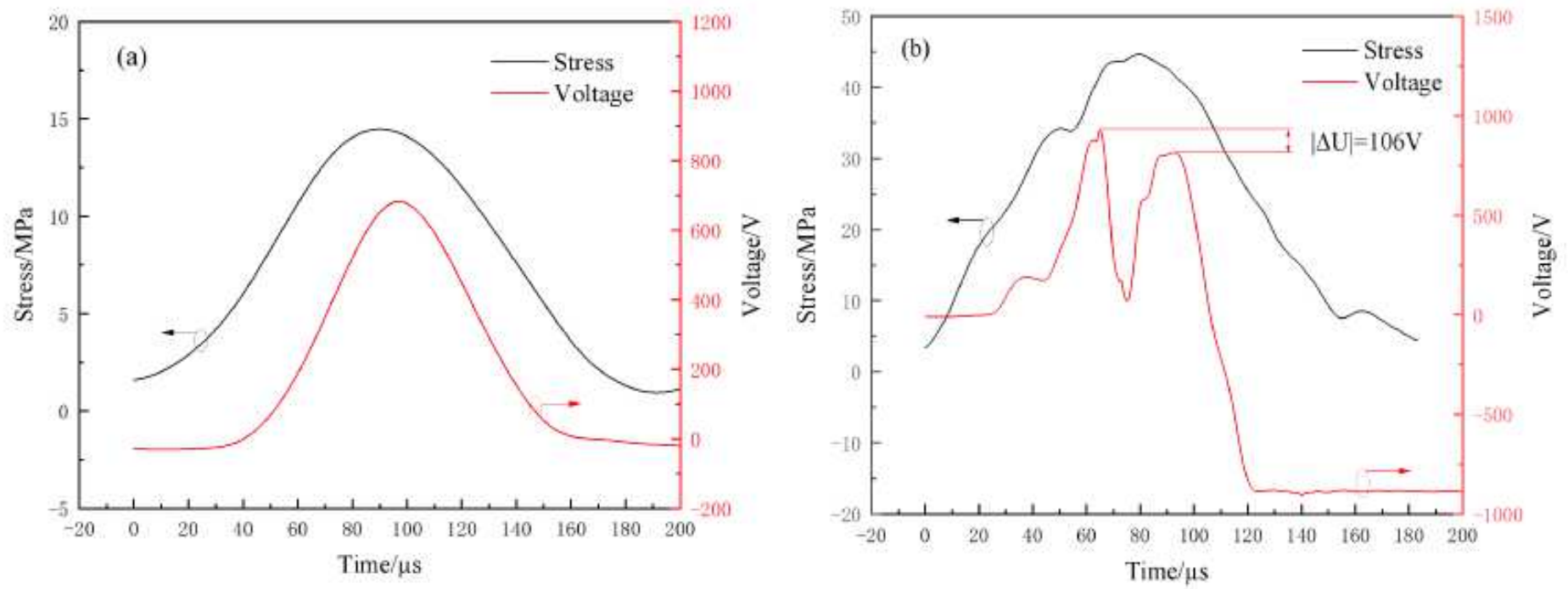

Figure 12

The time history curves of stress and output voltage at the temperature of 25囚 (a) The impact velocity $2.4 \mathrm{~m} / \mathrm{s}$, (b) The impact velocity $4.5 \mathrm{~m} / \mathrm{s}$
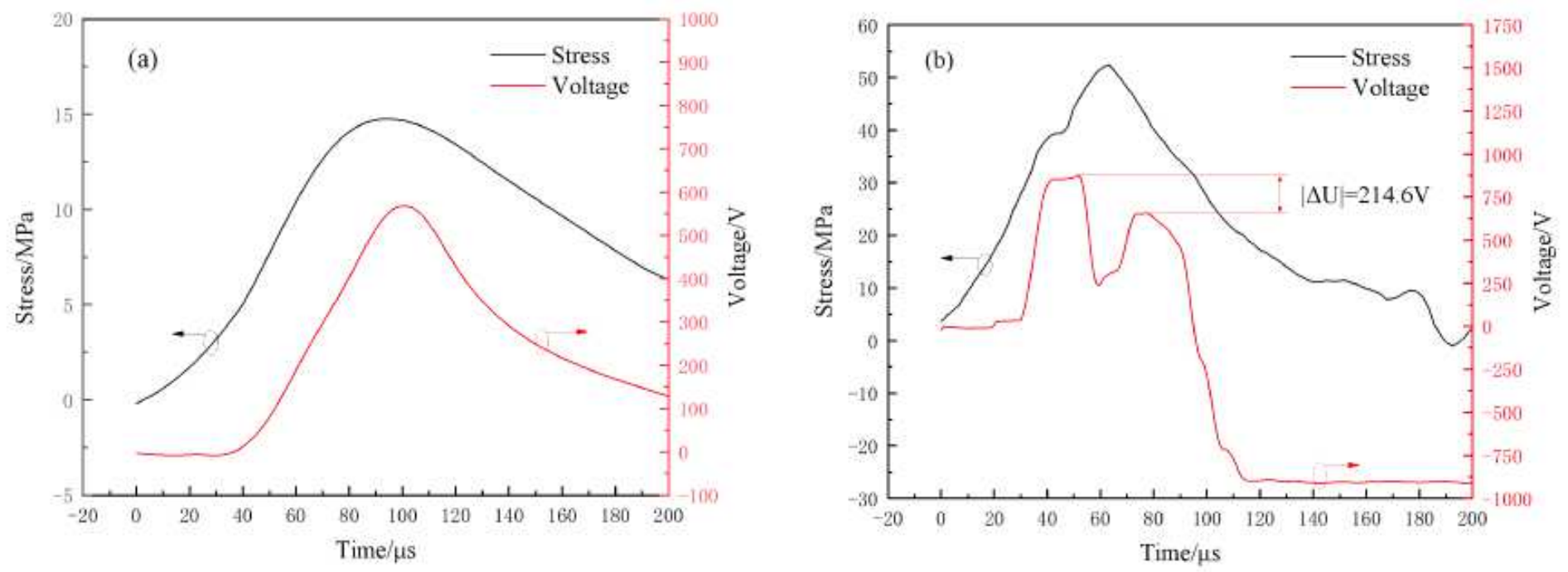

Figure 13

The time history curves of stress and output voltage at the temperature of $-40 \bigotimes$ (a) The impact velocity $2.4 \mathrm{~m} / \mathrm{s}$, (b) The impact velocity $4.5 \mathrm{~m} / \mathrm{s}$ 
(a)
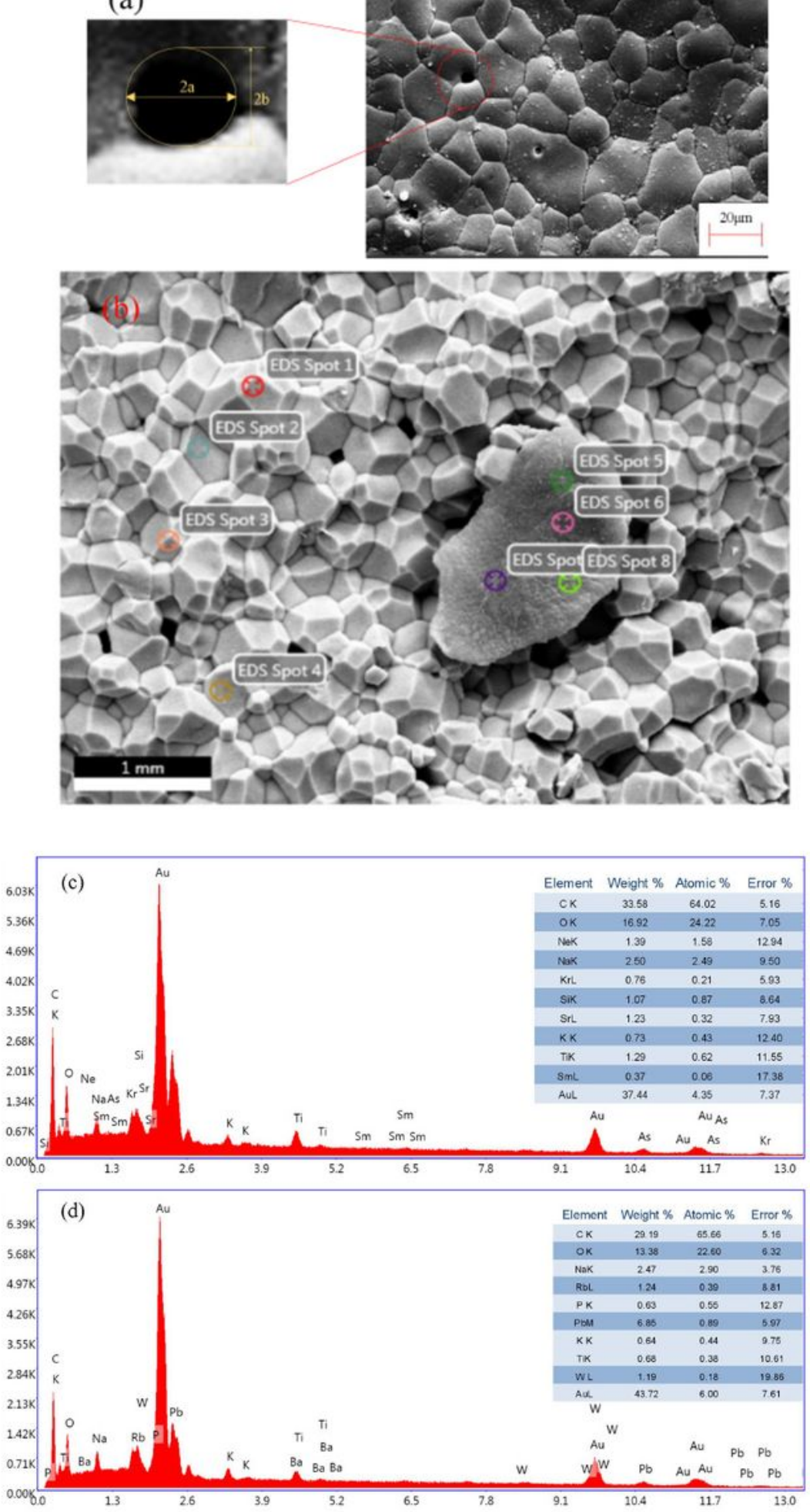

Figure 14

(a) The local SEM iconography of the piezoelectric ceramics (500x), (b) The local SEM iconography of the piezoelectric ceramics (5x), (c) Energy spectrum of EDS spot 7 of the local SEM iconography of the piezoelectric ceramics (5x),(d) Energy spectrum of EDS spot 8 the local SEM iconography of the piezoelectric ceramics $(5 x)$ 


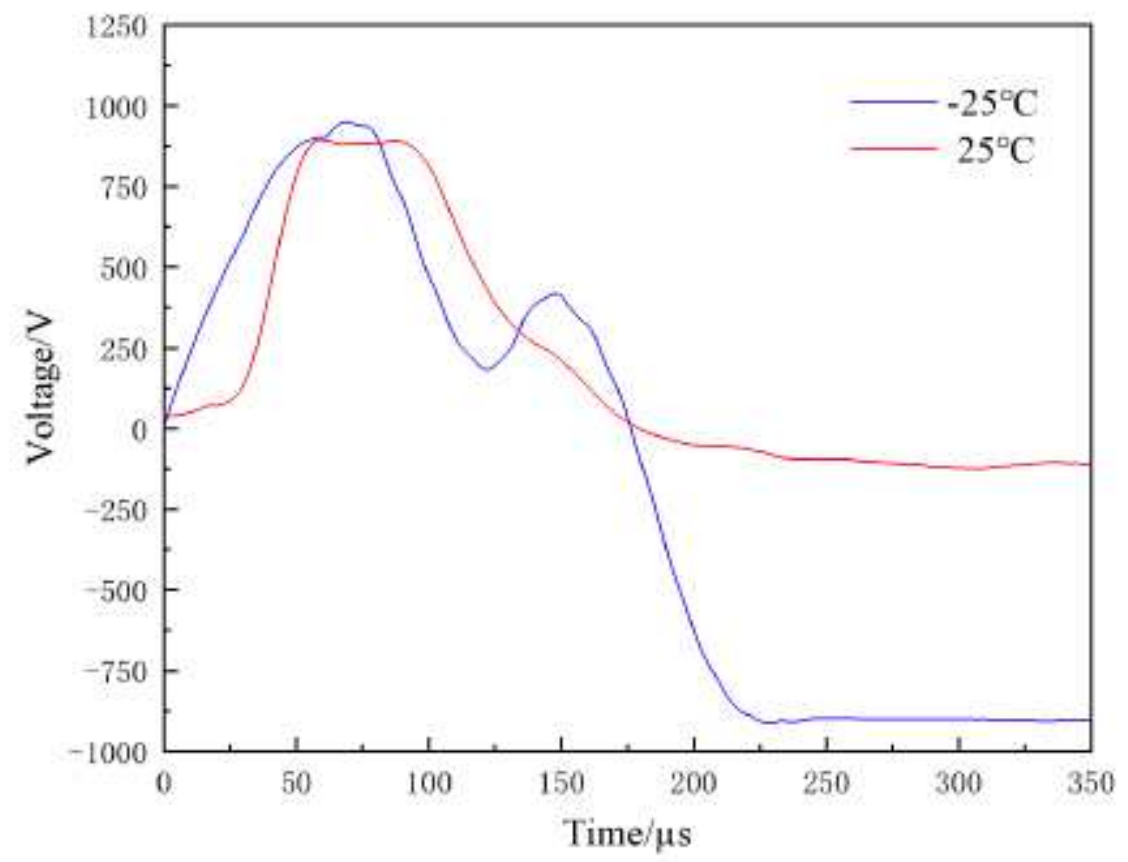

Figure 15

The voltage time history curve at impact speed of $3.4 \mathrm{~m} / \mathrm{s}$ at $-25 \rrbracket$ and $25 \rrbracket$

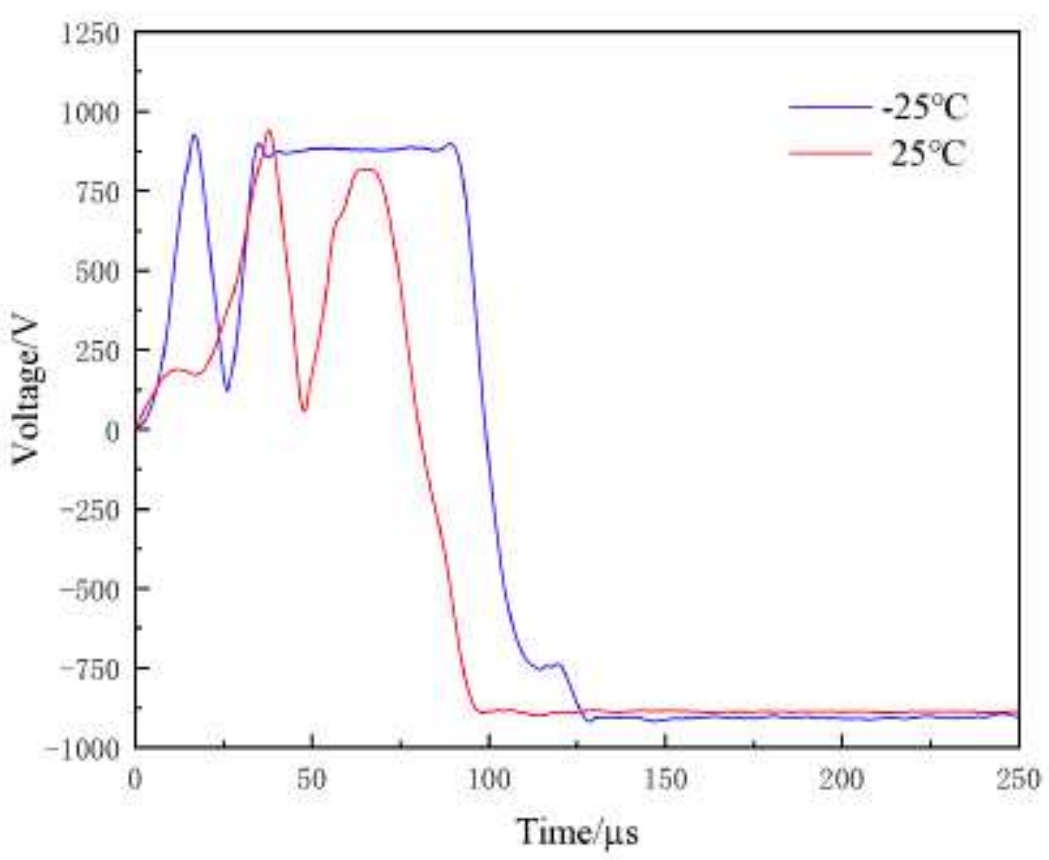

Figure 16

The voltage time history curve at impact speed of $4.5 \mathrm{~m} / \mathrm{s}$ at $-25 \rrbracket$ and $25 \rrbracket$ 

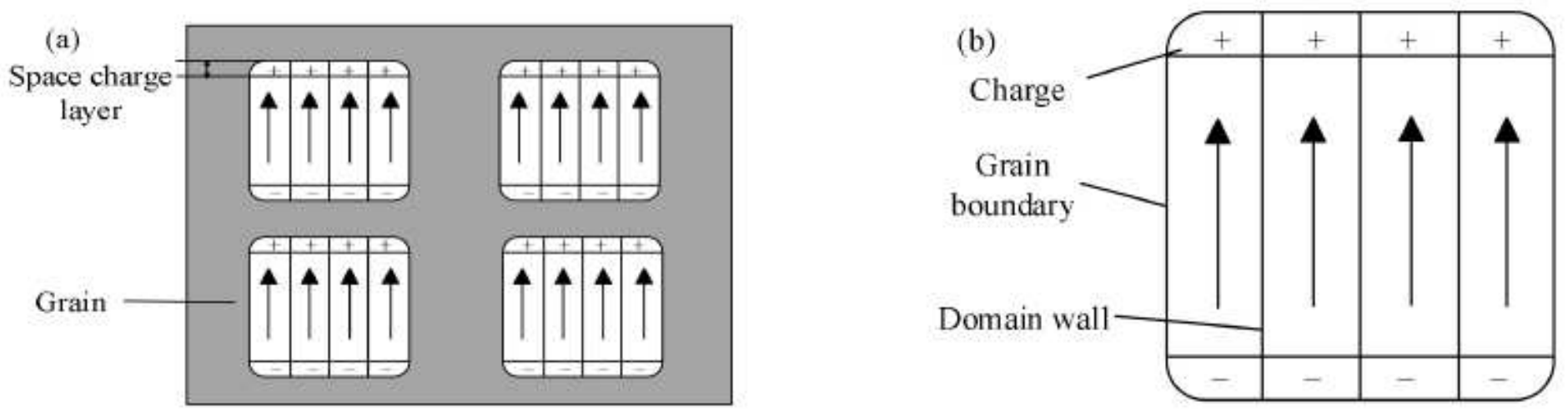

\section{Figure 17}

(a) The porous polarized grain structure, (b) The crystal domain structure of piezoelectric ceramics after polarization

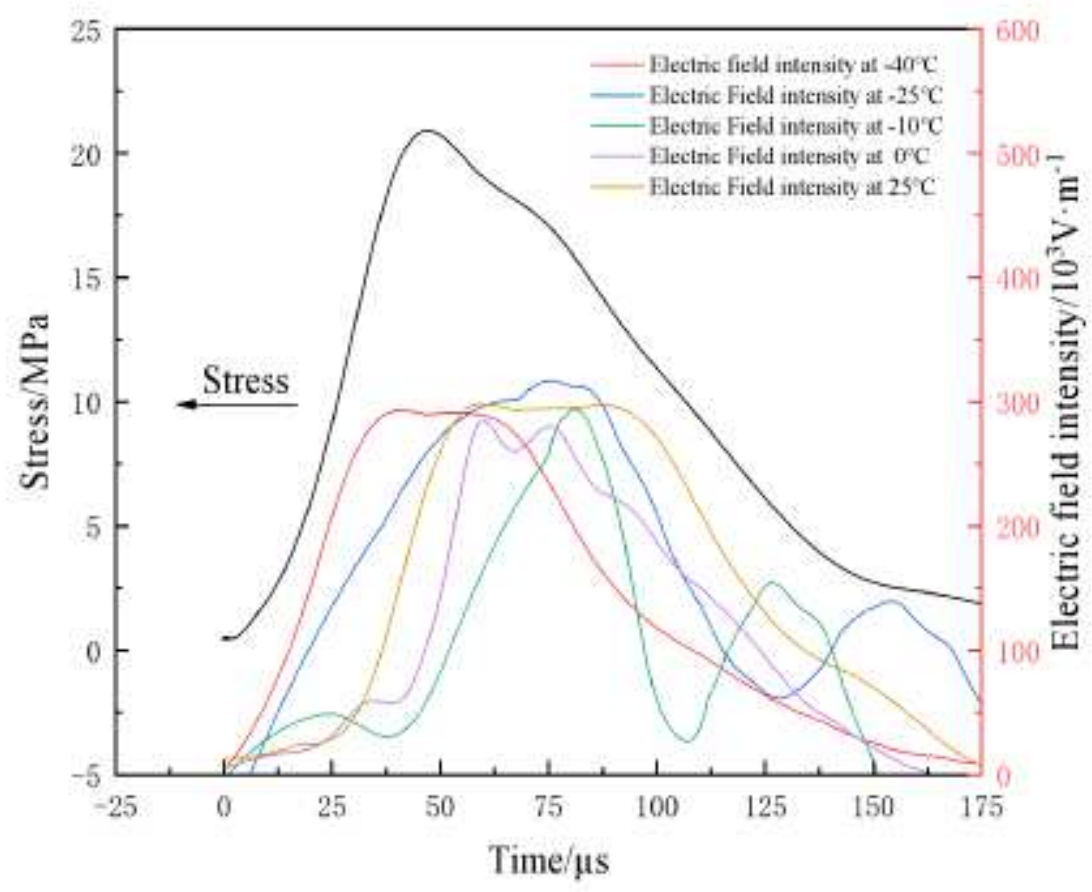

Figure 18

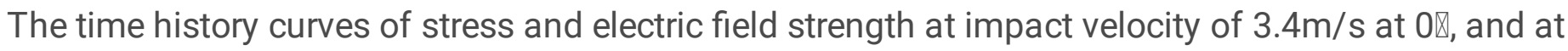
different temperatures 


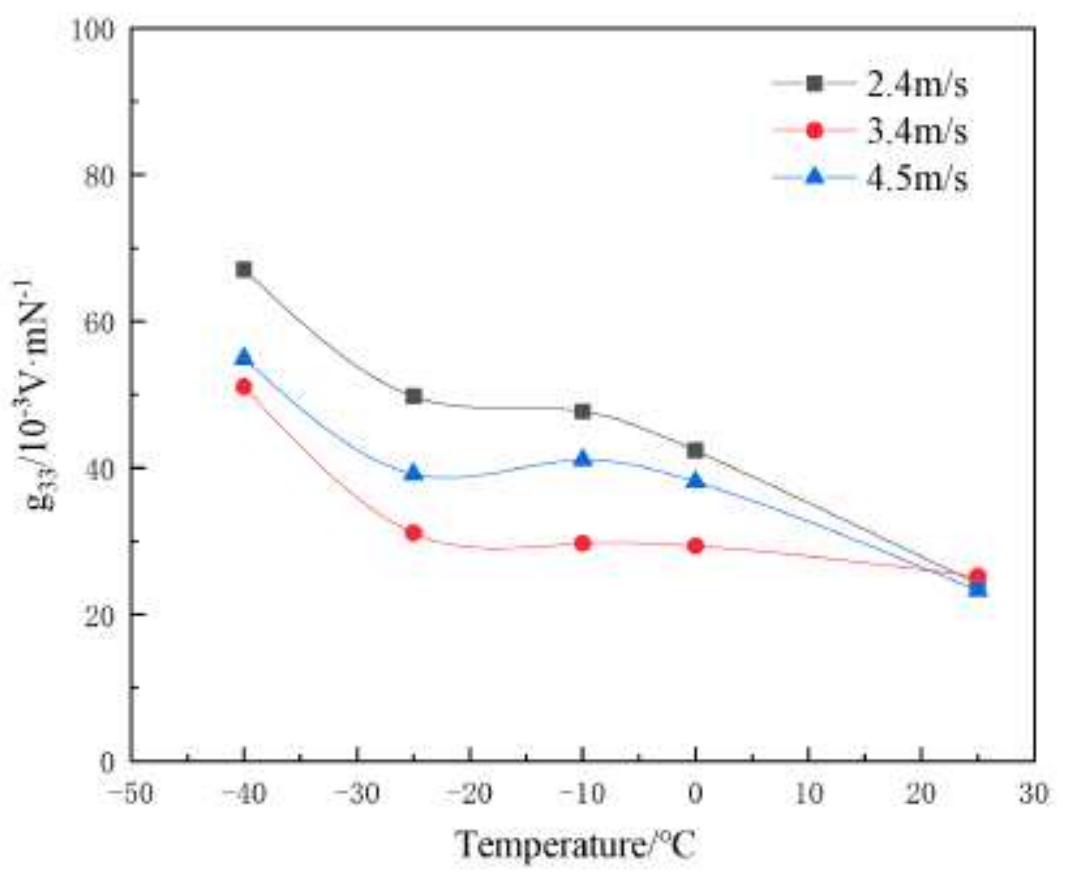

Figure 19

The relationship between piezoelectric voltage constant g33 and temperature at different impact velocities 